\title{
Maintien de l'ordre et maintien des ordres : les logiques spatiales du régiment des Gardes- françaises à Paris à la veille de la Révolution
}

\section{Clément Monseigne}

\author{
C OpenEdition \\ Journals \\ Édition électronique \\ URL : https://journals.openedition.org/cdg/6993 \\ DOI : $10.4000 /$ cdg.6993 \\ ISSN : 2107-7266 \\ Éditeur \\ UMR 245 - CESSMA \\ Référence électronique \\ Clément Monseigne, " Maintien de l'ordre et maintien des ordres : les logiques spatiales du régiment \\ des Gardes-françaises à Paris à la veille de la Révolution », Carnets de géographes [En ligne], 15 | 2021 , \\ mis en ligne le 30 avril 2021, consulté le 27 mai 2021. URL : http://journals.openedition.org/cdg/6993 \\ ; DOI : https://doi.org/10.4000/cdg.6993
}

Ce document a été généré automatiquement le 27 mai 2021.

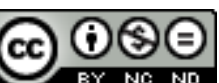

La revue Carnets de géographes est mise à disposition selon les termes de la Licence Creative Commons Attribution - Pas d'Utilisation Commerciale - Pas de Modification 4.0 International. 


\title{
Maintien de l'ordre et maintien des ordres : les logiques spatiales du régiment des Gardes-françaises à Paris à la veille de la Révolution
}

\author{
Clément Monseigne
}

\section{Introduction}

Le régiment des Gardes-françaises était sous l'Ancien Régime l'un des régiments les plus prestigieux de l'armée royale. Régiment d'élite, il était en effet affilié aux troupes de la Maison militaire du roi qui avaient la charge de la garde du souverain. Son importance était également visible sur les champs de bataille, puisqu'il était placé à la tête des armées dans les dispositifs tactiques. Son histoire militaire, cependant, prit fin en 1762, lorsqu'il livra ses derniers combats lors de la guerre de Sept Ans. Avec l'apaisement des conflits en Europe, les soldats aux Gardes-françaises, qui étaient casernés à Paris, représentèrent une charge considérable pour les finances de l'État, ce qui incita le lieutenant général de police à requérir leur soutien dans différentes tâches de maintien de l'ordre (Chagniot, 1985). Sous le règne de Louis XVI, les Gardesfrançaises représentaient théoriquement, en termes d'effectifs, la première force publique à Paris avec 3061 fusiliers et grenadiers, 72 canonniers, 114 tambours et instruments, 180 sergents et 198 officiers en $1789^{1}$. Ces nombres méritent néanmoins d'être pondérés puisqu'ils correspondent à l'effectif complet du régiment. Or, les Gardes-françaises fournissaient chaque semaine quatre compagnies ${ }^{2}$ pour monter la garde du roi à Versailles ainsi que des effectifs supplémentaires pour des missions ponctuelles auprès de la famille royale. Sur les effectifs restant à Paris, seule une partie était employée de façon permanente au maintien de l'ordre ; une lettre du colonel des Gardes-françaises, le maréchal de Biron, au contrôleur général des finances, Calonne, datée du 9 décembre 1785, indique que le régiment déployait quotidiennement un millier d'hommes pour la sûreté de Paris ${ }^{3}$. Outre le régiment des Gardes, trois autres 
unités militaires étaient chargées du maintien de l'ordre (Brouillet, 2002) : un détachement de 550 Gardes-suisses, 1400 hommes de la milice provinciale et 600 Invalides $^{4}$. Les effectifs des unités de police active ${ }^{5}$ étaient quant à eux plus modestes, la Garde de Paris fournissait 1200 fantassins et 265 cavaliers. On comptait également 300 Gardes de la ville, 200 hommes de la Robe courte et de la Connétablie et enfin 156 hommes des brigades de la Prévôté de l'île ${ }^{6}$ (Brouillet, 2008).

1 Paris à la fin du XVIII ${ }^{e}$ siècle était devenu, à l'issue d'un processus de centralisation, un centre politique majeur dans le royaume de France. "Qui tient Paris est maitre de tout le royaume et, pourrait-on ajouter, qui perd le contrôle de Paris perd le pouvoir " affirmait Jean Chagniot dans Paris et l'armée et au XVIII siècle (1985: 28). Le maintien de l'ordre à Paris à la fin de l'Ancien Régime était donc pour le pouvoir royal un enjeu politique central, qu'il devait s'assurer de maîtriser. Le contrôle de Paris était avant tout pour les unités qui en avaient la charge, un contrôle de l'espace et des populations, qui était mis en place à partir de points d'ancrage dans la ville tels que les casernes par exemple. Ce désir de surveiller et de réguler la ville dut néanmoins s'accommoder, dans la seconde moitié du XVIII ${ }^{\mathrm{e}}$ siècle, d'un développement des échanges et des flux, favorisé par l'amélioration significative des réseaux de circulation (Lepetit, 1988). Dès lors, l'objectif du pouvoir royal était «non plus fixer et marquer le territoire, mais laisser faire les circulations, contrôler les circulations, trier les bonnes et les mauvaises... » (Foucault, 2004: 67). Dans le cas de l'espace parisien à l'époque des Lumières, il s'agissait notamment de surveiller les flux croissants de voyageurs étrangers et de migrants (Roche, 2000 ; Blanc-Chaléard et al., 2001), la mendicité et le vagabondage (Romon, 1982), ainsi que les flux commerciaux qui étaient accompagnés de phénomènes de fraude et de contrebande (Abad, 2007). Ces différentes mobilités firent l'objet d'un contrôle étroit de la police parisienne, car « derrière de tels déplacements, les autorités de police redoutent de plus en plus l'absence de lien social organisé ou sa rupture. Pour tout dire, le désordre d'un monde mouvant et dépourvu de cadres " (Milliot et al., 2020 : 118-119). Le régiment des Gardes remplissait, pour sa part, des fonctions de contrôle très variées. Il avait notamment en charge la gestion des foules lors de réjouissances ou d'émeutes, ainsi que la garde des théâtres, mais les soldats étaient surtout astreints à effectuer des patrouilles quotidiennes en parcourant l'essentiel des rues de Paris. Destinées, en théorie, à surveiller les militaires dans la ville, elles finirent par prêter mainforte à la police parisienne (Chagniot, 1985). Les espaces et les populations qui étaient surveillés par les Gardes-françaises étaient donc très hétérogènes; de ce fait, les objectifs qui guidaient l'action du régiment dans le cadre du maintien de l'ordre semblent difficiles à cerner.

«Je voudrais vous "taquiner" un peu sur la définition de l'ordre. Je n'ai pas très bien compris quel ordre était remis en question ou quel était l'adversaire intérieur. À vous entendre, j'ai l'impression que c'est l'ordre public, je dirais même l'ordre politique. En fait cela suppose que le XVIII ${ }^{\mathrm{e}}$ siècle parisien ait été très agité [...] Et si je parle d'adversaire intérieur, c'est précisément à cause de cela, c'est que je ne vois pas qui est en face de vos Gardes-françaises et des toutes les autres institutions. Car le parlement parisien nous le prouve dix fois pour une, les commissaires nous le prouvent aussi : il n'y a pas de limites entre la délinquance, la petite criminalité et la création de désordres publics. » (Chagniot, 1974: 42)

Cette intervention de Robert Mandrou à l'issue d'une communication de Jean Chagniot, consacrée au maintien de l'ordre à Paris au XVIII ${ }^{\mathrm{e}}$ siècle, lors d'une séance de la Société d'histoire moderne et contemporaine, soulevait le problème de l'incertitude de la 
définition et des limites du maintien de l'ordre. Toute la difficulté était en effet de distinguer les multiples aspects des fonctions de police des Gardes-françaises, entre maintien de la sûreté publique et de l'ordre. Face à ce problème, une étude spatiale de l'action du régiment peut s'avérer utile pour comprendre ses objectifs et ainsi discerner ses différentes dimensions. Au-delà de l'action répressive à l'occasion de crises qui était évoquée par Robert Mandrou, il faut aussi s'interroger sur les autres aspects du maintien de l'ordre, qui permettaient au pouvoir royal de prévenir les troubles en amont.

Dans cette perspective, l'enjeu est d'affiner la compréhension des stratégies mises en œuvre par les agents du pouvoir royal à la fin de l'Ancien Régime pour contrôler Paris en observant leur traduction spatiale dans le détail. Le tracé de l'itinéraire des patrouilles du régiment des Gardes, l'emplacement de leurs casernes ou les dispositifs de contrôle des mobilités dont ils se chargeaient sont autant d'objets d'étude riches en enseignements sur le maintien de l'ordre à Paris à la fin du xvIII ${ }^{e}$ siècle. Leur restitution cartographique et leur analyse peuvent apporter des éléments utiles à l'éclairage de plusieurs questions: les stratégies du maintien de l'ordre, la perception de l'espace parisien et de ses populations par le pouvoir royal, mais également ses craintes et ses priorités.

4 Le lien entre espace et pouvoir développé par Michel Foucault (2004) a inspiré des travaux en géographie qui se sont intéressés à la territorialisation des stratégies de maintien de l'ordre avec des études de cas de villes comme Los Angeles (Herbert, 1996) ou Londres (Ogborn, 1993). Des approches identiques, portant sur la territorialisation du maintien de l'ordre dans les villes, se sont également développées en histoire. Le contrôle de l'espace urbain à travers sa mise en ordre a été très bien analysé par Brigitte Marin (1993) ou Vincent Milliot (2003) à travers l'étude du découpage administratif des villes de Naples et de Paris en quartiers de police. Reposant sur des représentations complexes (Germes, 2014), le découpage rationnel de l'espace en zones ou en quartiers constitue à lui seul une expression du pouvoir sur l'espace (Raffestin, 2019) et il dessine également un cadre à partir duquel se déploient des flux humains tels que les patrouilles, qui permettent la mise en œuvre pratique du contrôle de l'espace et de ses populations.

Les dispositifs employés pour le maintien de l'ordre ont fait l'objet d'une production scientifique abondante en sciences humaines. Le modèle de la patrouille et son influence sur les espaces criminogènes ont été étudiés en histoire, dans le cas de la maréchaussée par exemple (Gomez Pardo, 2012) et ont été largement analysés par les chercheurs nord-américains en criminologie (Sherman, Weisburd, 1995 ; Williams, Coupe, 2017). La problématique du contrôle des circulations dans la ville, évoquée par Foucault (2004), a également fait l'objet de travaux récents, avec une approche historique (Conchon, 2018). À l'échelle de la rue, la mise en œuvre pratique du maintien de l'ordre policier a été très bien étudiée par Vincent Milliot (2014) ou Nicolas Vidoni (2018), en revanche elle a été relativement négligée pour ce qui est du régiment des Gardes-françaises. L'étude des logiques spatiales de ce régiment est particulièrement intéressante en raison du lien très étroit qu'il entretenait avec l'autorité royale, et donc de la dimension politique que pouvait revêtir sa participation au maintien de l'ordre. Si l'implantation des casernes du régiment dans Paris a été développée dans les travaux de Jean Chagniot (1985), l'investissement de l'espace par les soldats aux Gardes dans le cadre des patrouilles ou des missions de maintien de l'ordre demeure à explorer. 
Les sources qui permettent d'étudier l'occupation de l'espace parisien par les hommes de cette unité ne manquent pas, le Service historique de la Défense à Vincennes conserve sous la cote GR Ya 272 de nombreux documents relatifs aux missions de maintien de l'ordre confiées au régiment des Gardes. Une série d'ordres donnés par l'état-major pour les patrouilles du régiment détaille notamment les itinéraires précis qu'elles empruntaient dans Paris. L'étude globale des trajets des différentes patrouilles des Gardes-françaises complète utilement les informations sur l'implantation des casernes et des postes du régiment en révélant des corrélations, des anomalies ou des logiques contradictoires entre ses points d'ancrage dans la ville et le tracé des patrouilles.

Une telle approche permet de soulever plusieurs questions concernant les dimensions spatiales du maintien de l'ordre : en quoi les logiques d'occupation de l'espace par le régiment des Gardes-françaises renseignent-elles sur la perception de la population parisienne par les agents du pouvoir royal ? Que disent-elles de ses inquiétudes et de ses priorités? Quelles stratégies mettait-il en œuvre et à quelles fins ? Un tel questionnement permet de s'interroger plus largement sur les objectifs du maintien de l'ordre dans la France de l'Ancien Régime, mais aussi sur ses limites.

La présence du régiment des Gardes dans Paris était, en premier lieu, matérialisée par leurs casernes, leurs postes fixes mais aussi par les logements de leurs officiers. Une approche géographique de ces lieux permettra de soulever le problème de la coexistence d'enjeux stratégiques avec des logiques financières et sociales dans l'implantation du régiment dans la ville. Les points d'ancrages des Gardes-françaises constituaient un réseau à partir duquel ils pouvaient sillonner l'espace urbain dans le cadre de leurs patrouilles. Si elles parcouraient la majorité des rues de Paris, l'étude détaillée de leur tracé révèle qu'elles étaient concentrées spatialement et temporellement dans les quartiers populaires qui constituaient des foyers de violence (Farge, 1979) et d'agitation. Les Gardes-françaises contribuaient donc à la fois à la sûreté publique et au maintien de l'ordre et il s'agira de comprendre, à travers le contrôle des mobilités, comment ces objectifs se distinguaient et s'articulaient dans le temps. L'enjeu sera enfin d'expliquer comment ces deux dimensions de l'action du régiment contribuaient chacune au maintien de l'autorité royale dans Paris.

\section{Une implantation stratégique dans la ville ? Les points d'ancrage du régiment des Gardes-françaises dans Paris à la fin de l'Ancien Régime}

\section{Un réseau de casernes encerclant Paris}

En 1764, à l'issue de la guerre de Sept Ans, le régiment des Gardes-françaises fut réorganisé par une grande réforme qui mit en place le casernement des soldats dans Paris (Chagniot, 1985). En 1788, à la veille de la Révolution, le régiment des Gardesfrançaises occupait seize casernes différentes dans Paris. Les casernes de la rue de Bourgogne, de la rue de Sève, de la rue du Faubourg Saint-Jacques, de la rue de l'Estrapade, de la rue Mouffetard, de la rue de l'Épée-de-Bois, de la rue de Bondy et de la rue Saint-Martin accueillaient chacune une compagnie. Celles de la rue Neuve-SainteGeneviève, de la rue du Faubourg-du-Temple et de la rue de la Pépinière logeaient chacune deux compagnies. Les casernes de la rue de Babylone, de la rue de Popincourt, 
de la rue Poissonnière et de la rue Verte accueillaient trois compagnies. La caserne de la rue de Loursine enfin pouvait loger quatre compagnies à cette époque.

À travers cette répartition, il est possible d'observer que l'implantation des Gardesfrançaises dans Paris était assez régulière : les casernes étaient implantées dans des quartiers populaires comme le faubourg Saint-Marcel (Burstin, 2005), mais également dans des quartiers aisés de l'Ouest parisien. Dans le cas du faubourg Saint-Honoré, cette présence peut être expliquée par la proximité du quartier des Porcherons, où se trouvaient de nombreux cabarets ${ }^{7}$. Ces lieux étaient en effet des sources de désordres potentiels dans l'espace parisien, puisque $29 \%$ des rixes et des disputes s'y déroulaient à la fin du XVIII ${ }^{e}$ siècle (Farge, 1979). En revanche la présence de trois casernes dans le faubourg Saint-Germain, où se trouvaient essentiellement des hôtels particuliers, peut sembler tout à fait étonnante. La construction de casernes dans les riches faubourgs pouvait être liée aux intérêts des propriétaires de biens immobiliers, qui étaient à la recherche d'un investissement rémunérateur comme l'a montré Jean Chagniot (1985). En effet, lorsque le casernement des Gardes-françaises fut décidé « le maréchal de Biron n'ayant aucun moyen de se procurer des fonds pour ces nouveaux établissements fit proposer à différents particuliers de faire bâtir des casernes suivant les plans que le Roi agréa et de les louer pour 27 ou 36 ans $»^{8}$. Le choix de l'emplacement des casernes a ainsi fait l'objet de négociations entre l'état-major et des propriétaires particuliers qui avaient tout intérêt à faire bâtir des casernes dans les riches faubourgs. Les écarts entre quartiers populaires et quartiers riches sont flagrants lorsque l'on observe les loyers perçus par ces propriétaires : la caserne de la rue de Bourgogne, située dans le faubourg Saint-Germain pouvait accueillir 140 hommes; elle était louée pour 4000 livres en 1771. Celle de la rue de Loursine pouvait elle aussi accueillir 140 hommes, cependant elle était située dans le faubourg Saint-Marcel et était louée pour 1500 livres seulement ${ }^{9}$. À l'automne 1789 , les propriétaires des casernes adressèrent un mémoire aux représentants de municipalité parisienne à l'occasion de la liquidation des biens du régiment des Gardes. Ils demandaient à être indemnisés, non sur la base de la valeur des bâtiments qu'ils louaient (600 000 livres), mais suivant les loyers qu'ils auraient dû toucher durant toute la durée des baux (923 650 livres) ${ }^{10}$, ce qui donne une idée des bénéfices qu'ils pouvaient espérer tirer de leurs investissements. L'implantation urbaine du régiment des Gardes-françaises était donc étroitement liée à des logiques financières et stratégiques puisque les casernes encerclaient Paris, en étant situées dans les faubourgs, aux marges de la ville. Les casernes ayant été construites à partir de 1764, leur situation dans Paris peut être expliquée par le manque de terrains appropriés au centre du tissu urbain qui était à cette époque encore structuré par l'urbanisme médiéval (Harouel, 1993 ; Ducoudray et al., 2000).

\section{Des officiers spatialement dissociés de leurs hommes}

Les officiers des Gardes-françaises ne résidaient pas dans les casernes, mais disposaient de logements particuliers qui étaient concentrés dans trois quartiers aisés de Paris : le quartier du Palais-Royal, le Marais et enfin le faubourg Saint-Germain ${ }^{11}$. Les deux colonels du régiment dans la deuxième moitié du xvIII ${ }^{e}$ siècle, le maréchal de Biron et le duc du Châtelet, résidaient tous deux dans le faubourg Saint-Germain, dans de somptueux hôtels particuliers : l'hôtel de Biron et l'hôtel du Châtelet. Lorsque l'on dresse la carte du logement des officiers dans Paris par grade, on constate que les officiers qui occupaient le sommet de la hiérarchie militaire, tels les capitaines, 
résidaient à proximité des officiers qui occupaient le bas de cette hiérarchie (voir figure 1). Cette hétérogénéité hiérarchique révèle une homogénéité sociale et indique que les officiers qui entraient dans le régiment au grade d'enseigne surnuméraire disposaient déjà d'un niveau de fortune élevé qui leur permettait de se mêler spatialement à leurs supérieurs. Il faut noter cependant la présence de plusieurs enseignes ${ }^{12}$ au dépôt des recrues du régiment, situé rue de la Chaussée-d'Antin, qui offrait la possibilité à ces officiers, essentiellement originaires de province (Chagniot, 1985), de loger à Paris à moindre coût.

Si les officiers ne logeaient pas avec leurs hommes, leurs carrières avaient cependant une influence déterminante sur le lieu de casernement des soldats. La répartition des compagnies dans les casernes était en effet décidée selon le bataillon auquel elles appartenaient. Les compagnies du premier bataillon, par exemple, étaient casernées dans le faubourg Saint-Germain. Or, l'affectation des compagnies à un bataillon dépendait de leur rang ${ }^{13}$, qui était défini en fonction de l'ancienneté des capitaines qui les commandaient ${ }^{14}$. De ce fait, les soldats aux Gardes-françaises furent fréquemment contraints de changer de caserne en raison d'un changement de capitaine à la tête de leur compagnie. En juin 1789, par exemple, les compagnies de Dumoncel et de Vaugiraud échangèrent de caserne ${ }^{15} \mathrm{car} \mathrm{M}$. de Vaugiraud s'avérait moins expérimenté que M. du Moncel ${ }^{16}$, ce qui modifiait le rang de leurs compagnies respectives. Cependant les deux compagnies n'échangèrent pas strictement de rang. Celui de troisième compagnie du quatrième bataillon dut être pourvu et de fil en aiguille, plusieurs autres compagnies ont pu voir leur rang et donc leur lieu de casernement affectés par la seule nomination de M. de Vaugiraud. Ainsi, à l'exception de la compagnie Colonelle qui bénéficiait toujours du premier rang, les compagnies des Gardes-françaises changèrent trois fois de caserne, en moyenne, entre 1780 et 1788 (voir tableau 1) ${ }^{17}$.

Tableau 1. l'échange de caserne des compagnies de Vaugiraud et de Dumoncel, un exemple de l'influence de l'ancienneté des officiers sur le lieu de résidence de leurs hommes ${ }^{18}$

\begin{tabular}{|c|c|c|c|c|}
\hline $\begin{array}{l}\mathrm{N}^{\circ} \text { de } \\
\text { compagnie }\end{array}$ & $\begin{array}{l}\text { Capitaine en avril } 1789 \\
\text { et date d'entrée dans le } \\
\text { régiment }\end{array}$ & $\begin{array}{l}\text { Rang } \\
\text { avril } 1789\end{array}$ & $\begin{array}{llr}\text { Capitaine } & & \text { en } \\
\text { juin } 1789 & \text { et } & \text { date } \\
\text { d'entrée } & \text { dans } & \text { le } \\
\text { régiment } & & \end{array}$ & Rang en juin 1789 \\
\hline 24 & $\begin{array}{l}\text { M. de Montesquiou } \\
\text { d'Artaignan (1750) }\end{array}$ & $\begin{array}{l}2^{e} \text { compagnie } d u \\
5^{e} \text { bataillon }\end{array}$ & Vaugiraud & $\begin{array}{l}\text { Dernière } \\
\text { compagnie } d u \\
4^{\mathrm{e}} \text { bataillon. }\end{array}$ \\
\hline 13 & M. du Moncel (1751) & $\begin{array}{l}3^{\mathrm{e}} \text { compagnie } \mathrm{du} \\
4^{\mathrm{e}} \text { bataillon }\end{array}$ & M. du Moncel (1751) & $\begin{array}{l}2^{\mathrm{e}} \text { compagnie } \mathrm{du} \\
5^{\mathrm{e}} \text { bataillon }\end{array}$ \\
\hline
\end{tabular}


Figure 1. Carte des domiciles déclarés par les officiers aux Gardes-françaises dans Paris en 1788, d'après le plan de Brion de la Tour

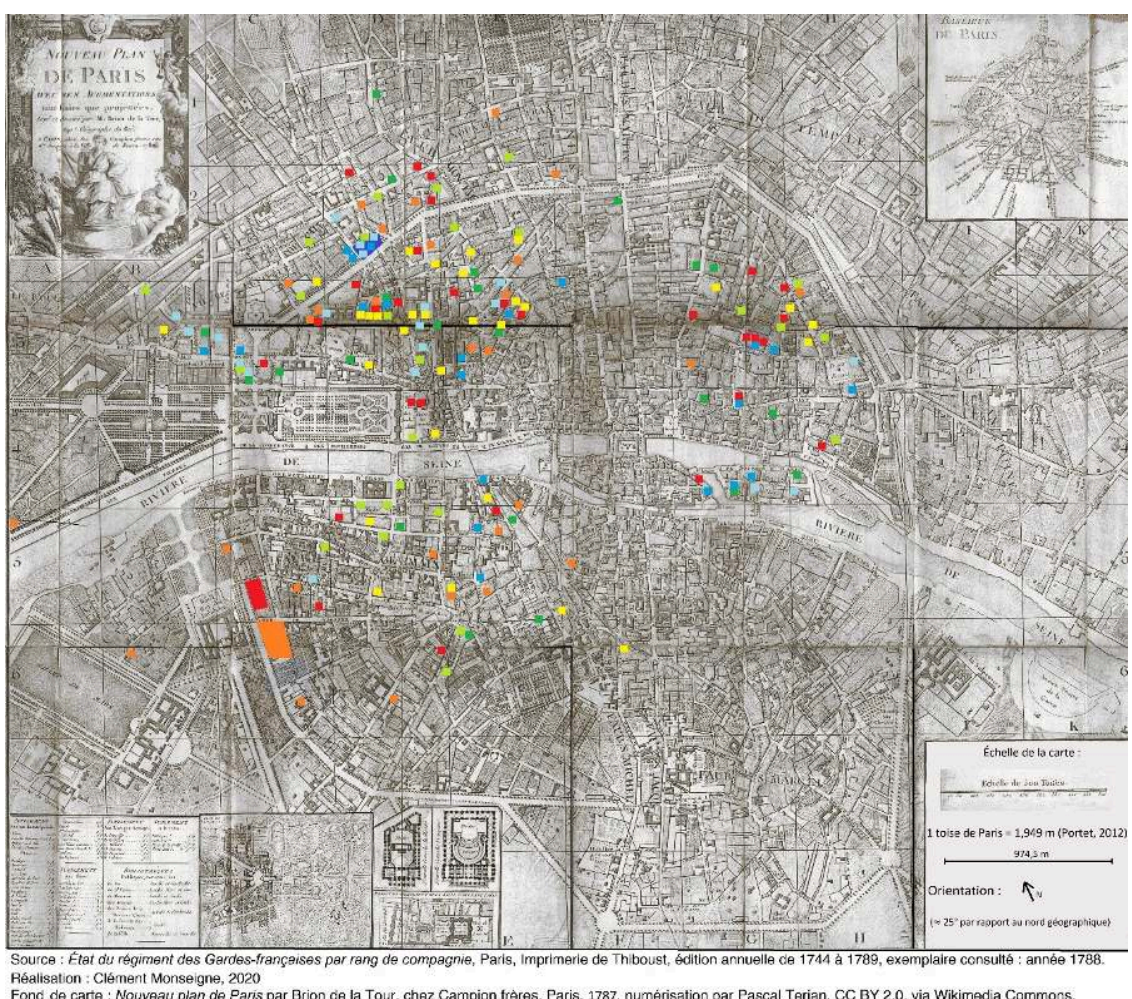

Source : État du régiment des Gardes-françaises par rang de compagnie, Paris, Imprimerie de Lamesle, édition annuelle de 1744 à 1789, exemplaire consulté : année 1788.

\section{Légende}

Domicile d'un capitaine

Domicile d'un lieutenant en premier

Domicile d'un lieutenant en second

Domicile d'un sous-lieutenant en premier

Domicile d'un sous-lieutenant en second

Domicile d'un enseigne

Domicile d'un enseigne surnuméraire

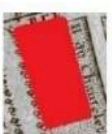

Hôtel du Châtelet

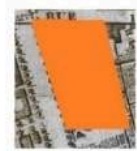

Hôtel de Biron

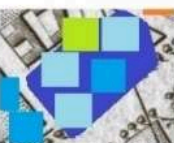

Dépôt des recrues

Au-delà du simple voisinage, la proximité spatiale des officiers allait parfois jusqu'à la cohabitation. En 1788, le capitaine Charles-François de Flavigny et son fils, Anne-JeanLouis, sous-lieutenant en premier de la compagnie de Froissard, résidaient dans un hôtel particulier situé 3 rue Gaillon qu'ils partageaient avec le Chevalier de la Bourdonnaye ainsi qu'avec Pierre-Gabriel-François de Lambilly et son cousin, le Chevalier Lambilly du Broutay ${ }^{19}$. Ce bâtiment occupait une surface de 147 toises carrées (Rittmann et Juinié, 1906), ce qui représente environ 550 mètres carrés. Les exemples 
de cohabitations, de voisinage et la concentration des officiers aux Gardes-françaises dans les quartiers bourgeois et aristocratiques sont tout à fait révélateurs de l'homogénéité sociale de ce corps.

Contrairement aux commissaires et aux inspecteurs de police qui logeaient majoritairement dans leur quartier d'affectation dans la deuxième moitié du XVIII ${ }^{\mathrm{e}}$ siècle (Milliot, 2014), les officiers aux Gardes ne résidaient pas nécessairement à proximité des compagnies qu'ils commandaient. Il est même plus fréquent d'observer une dissociation totale entre les lieux de résidence des officiers et les casernes de leur compagnie. En 1788, par exemple, tous les officiers de la compagnie de d'Ancour ${ }^{20}$ résidaient en rive droite de la Seine ou sur l'île de la Cité alors que la caserne de la compagnie se situait rue de Babylone en rive gauche ${ }^{21}$. Cette dissociation reproduisait en réalité la fracture entre officiers et soldats dans le régiment des Gardes-françaises, qui, à la fin de l'Ancien Régime, n'entretenaient guère de rapports durables, les soldats étant essentiellement encadrés par leurs bas-officiers (Corvisier, 1992).

Mais si les officiers ne dirigeaient pas les opérations de maintien de l'ordre, il a pu arriver, comme le racontait le libraire Hardy dans son journal, qu'ils aient joué un rôle de médiation lors d' " émotions populaires " ${ }^{22}$. Il affirmait, en effet, que le colonel du régiment s'était adressé, le 27 avril 1789, à une foule d'ouvriers qui souhaitait saccager la manufacture de Jean-Baptiste Réveillon. Ce manufacturier avait déclaré le 23 avril qu'il souhaitait voir baisser les taxes sur les grains et donc le prix du pain pour pouvoir diminuer le salaire de ses ouvriers et par conséquent le prix des objets manufacturés ; de cette déclaration, le bruit public ne retint que son intention de faire baisser les salaires, ce qui provoqua la colère des ouvriers parisiens qui souhaitaient s'en prendre à sa manufacture (Collot, 1934).

«M. le Duc du Châtelet colonel du régiment des Gardes-françaises les ayant pris par

la douceur et leur ayant parlé de manière à les calmer, était enfin parvenu à les diviser et à les renvoyer chacun chez eux ${ }^{23} »$.

Il est intéressant de noter que cette « émotion » ouvrière se déroula dans le faubourg Saint-Antoine, qui était, à cette époque, un point faible dans le maillage de Paris par les Gardes-françaises, étant donné qu'ils n'y disposaient que de deux corps de garde.

\section{Au-delà des casernes, la dispersion des bâtiments des Gardes- françaises dans Paris}

En dehors des casernes, le régiment disposait en effet de divers points d'ancrage dans Paris : le dépôt des recrues, qui servait à former et à sélectionner les jeunes gens aspirant à intégrer le régiment ; un hôpital réservé aux Gardes-françaises, situé dans le quartier du Gros-Caillou ; et trois postes de garde accueillant chacun une dizaine d'hommes, situés rue de la Jussienne, au Pont-Neuf, à l'abbaye Saint-Germain, et enfin deux autres dans le faubourg Saint-Antoine (Chagniot, 1985). Ces derniers postes furent créés à la suite de la dissolution en 1775 du corps des Mousquetaires noirs qui avaient en charge la sûreté de ce quartier. Les effectifs des postes de Gardes-françaises (neuf et douze hommes) étaient néanmoins modestes par rapport à ceux des Mousquetaires. Le régiment des Gardes-françaises avait aussi la charge de la garde des théâtres (Ravel, 1999), pour laquelle les soldats recevaient une indemnisation extraordinaire de quinze sols par représentation ${ }^{24}$, soit un peu plus que leurs appointements ordinaires quotidiens qui s'élevaient à dix sols ${ }^{25}$. Pour mener à bien cette mission, le régiment disposait, près des diverses salles de spectacle, à la Comédie-Française et à la ComédieItalienne, d'un corps de garde, sur le toit duquel un réservoir avait été installé pour 
permettre aux soldats d'intervenir en cas d'incendie. L'implantation urbaine du régiment des Gardes-françaises constituait finalement un dense réseau de casernes et de postes qui ceinturait Paris. Si l'on s'en tient à cette géographie des points d'ancrage du régiment, il serait possible de déduire, à l'instar de Jean Chagniot que « d'après la répartition des casernes en 1789 , les troupes de la Maison du roi n'étaient pas destinées particulièrement à tenir en respect les quartiers populaires de la capitale » (1985: 409). Cette affirmation mérite cependant d'être complétée puisqu'elle ne tient pas compte de la mobilité des soldats qui pouvaient se déployer à partir des postes et des casernes. Les patrouilles étaient en effet complémentaires des postes fixes, puisqu'elles permettaient une surveillance active du territoire urbain en le couvrant de façon régulière (Denys, 2003) ou en ciblant au contraire certains espaces.

\section{Le quadrillage de Paris par les patrouilles du régiment : une occupation méthodique de l'espace}

\section{De la police militaire à la police urbaine : les fonctions et l'organisation des patrouilles}

Les patrouilles du régiment des Gardes-françaises étaient composées de trois hommes seulement, un caporal et deux soldats qui portaient la baïonnette au bout du fusil. En comparaison, les patrouilles de la Garde de Paris étaient composées de six hommes ce qui semble indiquer une plus grande compétence des forces militaires dans le maintien de l'ordre. Le modèle militaire au siècle des Lumières était d'ailleurs reconnu pour son efficacité ; il influença l'organisation des forces de police qui se militarisèrent progressivement (Chagniot, 1985 ; Denys, 2008, Milliot et al., 2020). Toutefois, lorsqu'il s'agissait d'organiser des patrouilles pour lutter contre les contrebandiers aux portes de Paris (Abad, 2007), le marquis du Sauzay, major du régiment, affirmait au colonel que « des patrouilles contre les contrebandiers ne peuvent être moindres de dix hommes. Cette force est nécessaire pour qu'ils ne soient pas repoussés et même assassinés ${ }^{26}$. L'effectif réduit des patrouilles ordinaires, était donc tout juste suffisant pour maintenir le bon ordre dans une ville désarmée. Les patrouilles des Gardesfrançaises avaient lieu le soir, une première patrouille partait à huit heures du soir, $l^{\prime}$ heure de la retraite du régiment ${ }^{27}$, et parcourait les rues de Paris jusqu'à dix heures du soir. Elle était ensuite relevée par une seconde patrouille qui parcourait les rues jusqu'à minuit. Le marquis du Sauzay précisait que ces patrouilles, à l'origine, furent mises en place " pour arrêter les soldats en faute ${ }^{28}$, c'est-à-dire ceux qui transgressaient les horaires fixés pour les sorties hors des casernes, qui se trouvaient en état d'ébriété ou qui commettaient des désordres dans l'espace public ${ }^{29}$.

À partir du moment où l'appel était fait dans les casernes, une demi-heure après la retraite, les patrouilles des Gardes-françaises avaient ordre d'arrêter tous les soldats qui se trouvaient sur leur route et de les remettre à leur régiment d'affectation. Cette mission indique que les Gardes-françaises disposaient de prérogatives de police militaire ; ils remplissaient ainsi dans Paris les fonctions dont était chargée la maréchaussée en province (Brouillet, 1998). Les brigades de la Prévôté de l'île, rattachées à la maréchaussée (Gomez Pardo, 2012), étaient quant à elles peu impliquées dans la police militaire de Paris puisqu'elles étaient essentiellement déployées dans la banlieue de la ville et assuraient également la garde des barrières d'octroi. Les qualités de premier régiment de France et d'unité de la Maison militaire du roi permettaient 
aux Gardes-françaises d'exercer légitimement de telles fonctions et d'avoir une autorité sur les autres troupes. La légitimité des unités de la Maison militaire du roi dans les missions de police militaire était encore visible dans l'évolution des dispositifs de contrôle. La surveillance des militaires dans le quart nord-ouest de Paris, où se concentraient les cabarets, fut progressivement renforcée par des patrouilles sous l'autorité de la police, qui étaient composées d'un commissaire, d'un inspecteur de police (Vidoni, 2015), mais aussi d'un sergent des Gardes-françaises ou suisses (Chagniot, 1985).

Il conviendrait de compléter l'affirmation du marquis du Sauzay, car l'arrestation des soldats en faute n'était l'unique objet des patrouilles des Gardes qu'en théorie ; dans les faits, ces détachements « destinés en principe à faire respecter par les soldats la discipline militaire, ont fini par assumer les tâches les plus banales de police urbaine " (Chagniot, 1985 : 178). Ces patrouilles étaient en effet mentionnées dans le Plan de travail pour la sûreté de Paris de 1778 qui avait pour objet de coordonner l'action des différentes composantes de la force publique dans la ville (Milliot, 2016). Si l'action du régiment des Gardes venait épauler celle de la police parisienne, l'organisation et le commandement des patrouilles demeuraient entre les mains de l'autorité militaire. C'est en effet au commandement du régiment qu'il revenait de régler l'itinéraire et la durée des patrouilles, qui étaient ensuite communiqués « aux officiers de sûreté pour qu'ils puissent en conséquence régler leurs marches et opérations $»^{30}$. La coopération entre les Gardes-françaises et la police parisienne relevait donc davantage d'une répartition des tâches que d'une véritable action conjointe, d'autant plus qu'il existait des tensions sensibles entre les deux corps. Lors d'émotions populaires survenues à Paris, Hardy notait dans son journal, en date du 22 août 1787 que « les patrouilles du régiment des Gardes-françaises avaient été insultées en plusieurs endroits par des policiers qui les appelaient carpes au bleu ${ }^{31} \|^{32}$. Afin de mieux comprendre les logiques à l'œuvre dans l'organisation de ces patrouilles, une carte de l'itinéraire de ces dernières a été établie à partir du plan de Paris de Brion de la Tour. 
Figure 2. Carte des itinéraires des patrouilles du régiment des Gardes-françaises dans Paris en $1788^{33}$

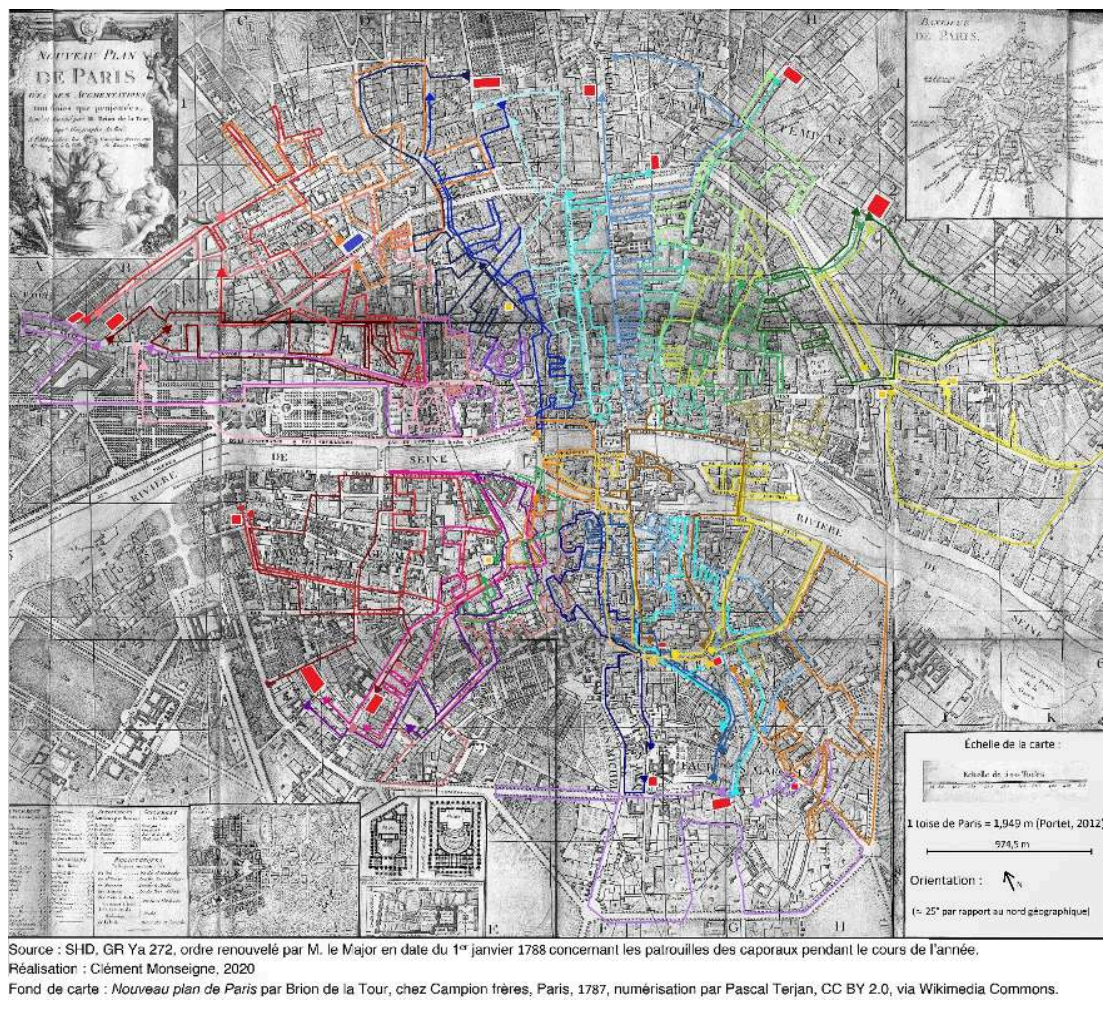

Légende

Itinéraires des patrouilles en rive gauche de la Seine
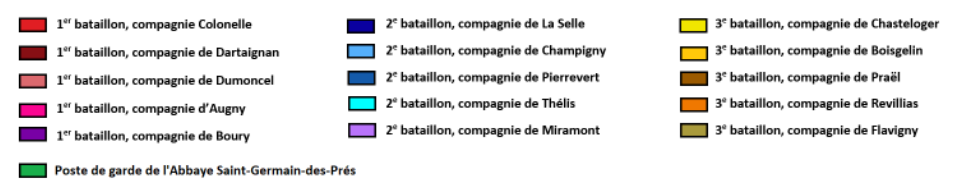

Itinéraires des patrouilles en rive droite et sur les îles de la Seine
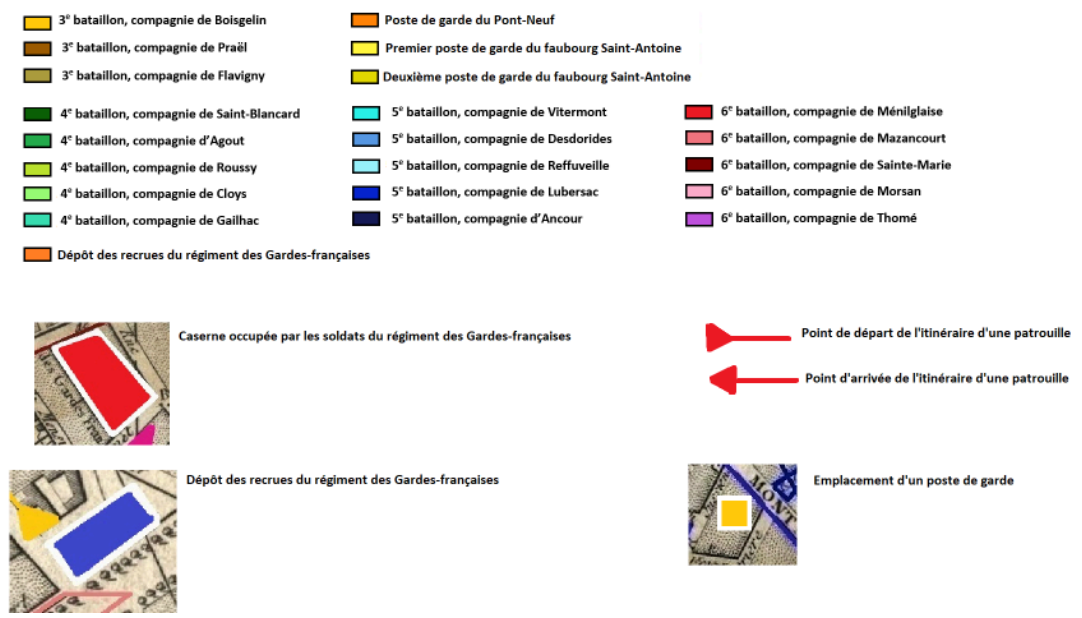

La figure 2 retrace les itinéraires des trente compagnies du régiment, de quatre postes de garde ${ }^{34}$, et des élèves du dépôt des Gardes-françaises, qui effectuaient eux aussi des patrouilles. La mission de police militaire confiée au régiment pose question lorsque l'on voit que le quartier des Porcherons, qui abritait de nombreux cabarets, était en partie parcouru par la patrouille du dépôt des recrues. Les élèves du dépôt recensés en 
1782 avaient entre 9,5 ans et 20 ans, leur moyenne d'âge était de 14,5 ans ${ }^{35}$. Il est ainsi assez difficile d'envisager que des soldats ivres du régiment des Gardes ou d'autres régiments aient accepté de suivre docilement ces jeunes recrues. Plus généralement, il serait même intéressant de se demander quelle attitude pouvaient avoir les soldats des patrouilles lorsqu'ils devaient arrêter un de leurs camarades en faute dans un cabaret : les exigences du service pouvaient-elles supplanter les solidarités militaires nouées dans les casernes ? La vie commune des soldats dans ces espaces clos contribuait en tout cas à les rendre étrangers à la population civile qu'ils surveillaient dans le cadre de leur service (Chagniot, 1985).

\section{Des patrouilles concentrées dans l'espace populaire parisien}

Le trajet des patrouilles suivait globalement la géographie du Paris populaire. Les compagnies situées dans les faubourgs aisés comme le faubourg Saint-Germain se dirigeaient en priorité vers l'est et donc vers les quartiers populaires ; les compagnies situées dans les quartiers populaires ne se dirigeaient pas vers les riches faubourgs, elles quadrillaient minutieusement le faubourg Saint-Marcel ou le quartier des Halles. Les compagnies stationnées dans le faubourg Saint-Germain, au contraire, délaissaient un certain nombre de voies publiques, comme le quai d'Orsay, la rue de Poitiers ou la rue des Saints-Pères. Le facteur démographique ne suffit pas à expliquer le tracé des patrouilles étant donné que le faubourg Saint-Marcel ne faisait pas partie des quartiers les plus densément peuplés à la fin de l'Ancien Régime (Karéiev, 1912). Les logiques spatiales des patrouilles révèlent donc avant tout une volonté de maîtrise de l'ordre public dans l'espace populaire parisien, qui constituait un espace suspect.

L'attention portée aux quartiers populaires est également visible lorsque l'on observe les distances parcourues par les patrouilles des différentes compagnies du régiment (voir tableau 2). La patrouille de la compagnie de Ménilglaise, qui inspectait le riche faubourg Saint-Honoré, parcourait dix kilomètres en deux heures. La patrouille de la compagnie de Vitermont, quant à elle, ne parcourait que cinq kilomètres durant ce même laps de temps. Elle était cantonnée à l'espace situé entre la rue Saint-Denis et la rue Saint-Martin, quartier modeste parmi les plus densément peuplés de la ville (Ducoudray et al., 2000). Cette tendance est également visible à l'échelle des bataillons : les patrouilles du sixième bataillon, chargées de surveiller le faubourg Saint-Honoré et le quartier du Louvre parcouraient en moyenne huit kilomètres et demi contre cinq kilomètres six cents mètres pour celles du deuxième bataillon, affectées au faubourg Saint-Marcel. « C'est le quartier où habite la populace de Paris, la plus pauvre, la plus remuante et la plus indisciplinable. Il y a plus d'argent dans une seule maison du faubourg Saint-Honoré que dans tout le faubourg Saint-Marcel, ou Saint-Marceau pris collectivement » écrivait Louis-Sébastien Mercier dans son Tableau de Paris ${ }^{36}$. Cette association entre les classes populaires et les classes dangereuses, pour l'ordre public et pour l'ordre politique, était, selon toute vraisemblance, partagée par les autorités civiles et militaires responsables du maintien de l'ordre à Paris. La distance épargnée aux soldats qui patrouillaient dans les quartiers populaires, et donc le temps supplémentaire qui leur était accordé, devaient leur permettre d'inspecter ces espaces avec une plus grande minutie. Ils étaient en effet censés consacrer l'intégralité du temps qui leur était imparti aux missions de surveillance et de maintien de l'ordre comme l'indiquaient les consignes du commandement « il est ordonné aux sergents de garde aux casernes et aux postes de faire partir les patrouilles à l'heure prescrite et de 
veiller qu'elles ne rentrent pas avant l'heure dite $»^{37}$. Le plan établi pour les patrouilles du régiment des Gardes-françaises associait deux paramètres déterminants pour l'efficacité du dispositif de contrôle : la fréquence des passages sur le terrain et leur durée (Williams, Coupe, 2017). Ainsi, comme l'affirme Claude Raffestin « tout réseau révèle, au même titre que les maillages et l'implantation des points, une certaine maîtrise de l'espace, en fait une maîtrise de l'enveloppe spatio-temporelle » (2019: 150).

Tableau 2 : distances parcourues par les patrouilles des différentes compagnies du régiment des Gardes-françaises en $1788^{38}$

\begin{tabular}{|l|l|l|l|l|l|}
\hline \multicolumn{2}{|l|}{ Premier bataillon } & \multicolumn{2}{l}{ Deuxième bataillon } & \multicolumn{2}{l|}{ Troisième bataillon } \\
\hline Compagnie & Distance & Compagnie & Distance & Compagnie & Distance \\
\hline Colonelle & $6,0 \mathrm{~km}$ & Laselle & $4,8 \mathrm{~km}$ & Chasteloger & $5,0 \mathrm{~km}$ \\
\hline Dartaignan & $5,3 \mathrm{~km}$ & Champigny & $5,3 \mathrm{~km}$ & Boisgelin & $4,9 \mathrm{~km}$ \\
\hline Dumoncel & $6,3 \mathrm{~km}$ & Pierrevert & $5,2 \mathrm{~km}$ & Praël & $5,4 \mathrm{~km}$ \\
\hline D’Augny & $7,3 \mathrm{~km}$ & Thélis & $5,1 \mathrm{~km}$ & Revillias & $7,9 \mathrm{~km}$ \\
\hline Boury & $6,3 \mathrm{~km}$ & Miramont & $7,7 \mathrm{~km}$ & Flavigny & $6,6 \mathrm{~km}$ \\
\hline Quatrième bataillon & Cinquième bataillon & Sixième bataillon \\
\hline Compagnie & Distance & Compagnie & Distance & Compagnie & Distance \\
\hline Saint-Blancard & $5,3 \mathrm{~km}$ & Vitermont & $5,1 \mathrm{~km}$ & Ménilglaise & $10 \mathrm{~km}$ \\
\hline D’Agout & $6,1 \mathrm{~km}$ & Desdorides & $7,9 \mathrm{~km}$ & Mazancourt & $9,1 \mathrm{~km}$ \\
\hline Roussy & $6,2 \mathrm{~km}$ & Reffuveille & $6,8 \mathrm{~km}$ & Sainte-Marie & $6,2 \mathrm{~km}$ \\
\hline De Cloys & $4,9 \mathrm{~km}$ & Lubersac & $7,8 \mathrm{~km}$ & Morsan & $7,3 \mathrm{~km}$ \\
\hline Gailhac & $6,7 \mathrm{~km}$ & D'Ancour & $7,0 \mathrm{~km}$ & Thomé & $10 \mathrm{~km}$ \\
\hline Dépôt des recrues et postes de garde & Distance & Postes de garde & Distance & Postes de garde & Distance \\
\hline & $8,1 \mathrm{~km}$ & Abbaye St-Germain & $3,6 \mathrm{~km}$ & Faub. St-Antoine 1 & $3,9 \mathrm{~km}$ \\
\hline Dépôt des recrues & Pont-Neuf & $3,2 \mathrm{~km}$ & Faub. St-Antoine 2 & $4,8 \mathrm{~km}$ \\
\hline & & & & \\
\hline
\end{tabular}

\section{Prévenir le crime et la sédition : le rôle dissuasif des patrouilles dans les quartiers populaires}

La difficulté à lire l'itinéraire des patrouilles est, elle aussi, révélatrice : dans les quartiers populeux et populaires, les trajets se multipliaient et s'entrecroisaient alors qu'ils sont plutôt isolés dans les quartiers plus aisés. Ainsi, six compagnies tournaient autour de l'abbaye Sainte-Geneviève alors que les rues du faubourg Saint-Germain étaient rarement empruntées par plus de deux compagnies. Les passages successifs de patrouilles différentes sur des axes identiques (Denys, 2003), en dépit d'effectifs modestes, renforçaient le contrôle des espaces populaires qui étaient également des espaces criminogènes. En effet, les rues du quartier Maubert situées entre la rue SaintJacques et la rue des Bernardins, en rive gauche de la Seine, ont été identifiées par 
Arlette Farge comme un espace où se concentraient les actes de violence (Farge, 1979). Une comparaison du trajet des patrouilles du régiment des Gardes-françaises avec la carte des lieux où se sont déroulés des faits de violence entre 1760 et 1785, établie par Arlette Farge et André Zysberg (Farge, 1979), permet d'observer que les patrouilles qui quadrillaient les quartiers fortement touchés par la violence, comme le quartier Maubert, empruntaient des itinéraires sinueux qui obligeaient les soldats à revenir sur leurs pas à de multiples reprises.

Il est néanmoins étonnant de constater que certaines patrouilles évitaient des rues, qui étaient pourtant situées sur leur trajet et de surcroît dans des quartiers touchés par la violence à l'instar de la rue Saint-Fiacre, délaissée par les patrouilles. La rue SaintFiacre, au droit de laquelle passait la patrouille de la compagnie de Lubersac, se situait en effet près de la rue Montmartre, dans laquelle Arlette Farge avait pu recenser à la fois de nombreux actes de violence et des domiciles de prévenus (Farge, 1979). Cette anomalie était en réalité liée à l'existence d'un contrôle privé de cet espace ; la rue Saint-Fiacre ou rue du Figuier fut en effet fermée par une grille en 1714-1715, sur la demande de ses habitants (Vidoni, 2018). Cette grille, dont la clé était détenue par les riverains, permettait d'isoler la rue du Figuier des boulevards, où circulaient de nombreux individus turbulents (Turcot, 2005). L'évitement de cette rue par la patrouille de la compagnie de Lubersac, confirme bien que la construction de la grille était une manière pour les riverains d'échapper à la surveillance étatique, comme l'a affirmé Nicolas Vidoni (2018).

Dans d'autres villes européennes, comme à Genève, certains militaires pouvaient être dépouillés de leurs armes et de leurs uniformes lors des patrouilles afin de lutter plus efficacement contre le crime (Cicchini, 2009). Les soldats aux Gardes-françaises jouaient donc plutôt un rôle dissuasif dans la sûreté de Paris par leur présence, en armes et en uniformes, dans les « théâtres de la violence ", à des heures où se concentraient les actes de violence (Farge, 1979). Afin de compléter cette mission, les patrouilles ordinaires du régiment étaient renforcées par des « patrouilles grises » qui comportaient des soldats aux Gardes en civil, et qui étaient bien plus efficaces, comme le rappelait Jean Chagniot « seuls les naïfs se laissent surprendre par les patrouilles trop bruyantes » (1988:40). Le modèle routinier de la patrouille offrait aux soldats aux Gardes-françaises la possibilité d'acquérir une expérience précieuse, qui leur permettait, à l'issue des huit années de leur engagement dans le régiment, d'être utiles à la police de la ville. En effet : « la Lieutenance recruta d'anciens soldats, rompus aux méthodes de quadrillage de l'espace afin de mieux l'occuper " (Vidoni, 2015 : 470-471). Mais au-delà des méthodes de surveillance, les Gardes-françaises pouvaient aussi se familiariser avec une grande partie de l'espace parisien au cours de leurs patrouilles, étant donné que les compagnies changeaient fréquemment de caserne comme nous l'avons expliqué plus haut. Les itinéraires de patrouilles étant attachés, non aux compagnies, mais aux casernes ${ }^{39}$, les soldats aux Gardes-françaises n'étaient pas cantonnés à la surveillance d'un quartier particulier. Ils pouvaient donc être amenés, au cours de leur carrière, à patrouiller dans de riches faubourgs puis dans des quartiers populaires. Tout en consolidant l'expérience des soldats dans le quadrillage de l'espace, cette rotation régulière, associée à leur casernement, contribuait à distendre les liens qu'ils pouvaient tisser avec la population d'un quartier.

Les trente patrouilles de Gardes françaises, cependant, ne parvenaient pas à scruter l'ensemble des rues de l'espace parisien. À quelques pas de la rue Saint-Fiacre, les rues perpendiculaires à la rue Beauregard, dans le quartier de Bonne-Nouvelle n'étaient 
parcourues par aucune patrouille du régiment. L'absence de patrouille dans ces rues peut être expliquée par leur tracé particulier, qui aurait très certainement sensiblement allongé leur temps de trajet. Cet espace n'était cependant pas totalement délaissé par le pouvoir royal puisque les rues qui n'étaient pas parcourues par la Garde de Paris ou les Gardes-françaises étaient scrutées par les commissaires et les inspecteurs qui les parcouraient en carrosse, et leurs auxiliaires, à pied (Vidoni, 2015). Par ailleurs, il convient de noter qu'en matière de maintien de l'ordre, les préoccupations de la police parisienne et celles du commandement des Gardesfrançaises ne se rejoignaient pas nécessairement en tous points. Il est par exemple assez difficile d'établir une corrélation entre les lieux de mendicité (Romon, 1982) et le trajet des patrouilles du régiment. Les Gardes-françaises ne semblaient pas non plus prêter d'attention particulière au faubourg Saint-Germain, où résidaient la majorité des étrangers fichés par la police à la fin de l'Ancien Régime (Roche, 2000).

La géographie des patrouilles du régiment des Gardes-françaises révèle le rôle dissuasif de cette unité dans le cadre du maintien de l'ordre public et politique, au-delà de la mission de police militaire qui leur était originellement confiée. Il s'agissait pour le pouvoir royal de matérialiser son autorité sur l'ensemble de la ville, en insistant particulièrement sur les espaces populaires qui constituaient de potentiels foyers d'agitation. L'action des Gardes-françaises s'inscrivait en cela dans un mouvement plus large : l'émergence au XvIII siècle, en France, mais aussi en Italie par exemple, d'une volonté étatique de « disciplinement matériel du territoire $»^{40}$ (Alessi, $2002: 33$ ) par l'intermédiaire d'agents distincts de la force publique municipale, traditionnellement en charge du maintien de l'ordre. Le contrôle régulier de Paris assuré par les patrouilles constituait une première modalité d'exercice de l'autorité royale sur le territoire urbain et ses populations. Néanmoins, la régularité spatiale et temporelle des patrouilles, ainsi que leur mobilité constante, faisaient échapper à leur surveillance une grande partie des dynamiques du phénomène urbain. Ce sont au contraire des dispositifs statiques, auxquels les Gardes-françaises contribuaient occasionnellement, qui permettaient de maîtriser les mobilités dans l'espace urbain.

\section{Le contrôle et la mise en ordre des mobilités dans l'espace parisien par les Gardes-françaises}

\section{Un concours profitable à la sûreté publique}

La police des transports dans la France d'Ancien Régime était ordinairement confiée à la police du roulage. Elle avait pour mission de faire appliquer la réglementation relative aux véhicules qui transportaient des hommes ou des marchandises, afin de préserver la sûreté publique ainsi que l'état des routes (Conchon, 2018). La nécessité d'une gestion de la circulation des carrosses à Paris constituait un enjeu majeur de sûreté publique lors de grands rassemblements dans la ville. La bousculade mortelle, qui eut lieu près de la place Louis XV, le 30 mai 1770 lors du feu d'artifice tiré à l'occasion du mariage du Dauphin et de Marie-Antoinette, fut selon Louis-Sébastien Mercier « occasionnée par la foule des voitures qui obstruèrent la rue, unique passage ouvert à l'affluence prodigieuse du peuple qui se portait en foule à la triste illumination des boulevards $»^{41}$. Afin d'éviter de tels drames, le Guet et le régiment des Gardesfrançaises étaient mobilisés pour empêcher les embarras des carrosses. Le 30 mai, cependant, le régiment des Gardes ne fut pas requis. Le comte de Ségur, dans ses 
mémoires, imputa cette absence à la mesquinerie du Prévôt des Marchands qui aurait refusé de payer le supplément de solde réclamé par le maréchal de Biron ${ }^{42}$.

Afin d'éviter qu'un tel drame ne se reproduise, les soldats furent sollicités en août 1775, lorsque l'ambassadeur de Sardaigne à Paris donna une somptueuse fête à l'occasion du mariage entre le prince de Piémont et la princesse Clotilde de France. Les festivités eurent lieu dans le Vauxhall ${ }^{43}$ nouvellement construit sur le boulevard du Midi, et les invités furent conviés à un feu d'artifice qui devait se dérouler à onze heures du soir puis à un bal. Pour cela, un dispositif conséquent fut mis en place, 41 sergents, 43 caporaux et 418 soldats furent répartis en huit postes autour du Vauxhall (voir figure 3$)^{44}$. Les soldats étaient chargés de régler la circulation des voitures, mais ils n'avaient pas pour mission de restreindre l'accès des piétons au boulevard du Midi, ces derniers étant libres d'aller et venir à leur guise. La reconstitution du plan de circulation établi à l'occasion de la fête donnée par l'ambassadeur de Sardaigne permet de mieux comprendre les objectifs du contrôle des mobilités à travers sa mise en œuvre sur le terrain.

Figure 3. Plan de circulation établi à l'occasion de la fête donnée par l'ambassadeur de Sardaigne sur le boulevard du Midi le 25 août 1775

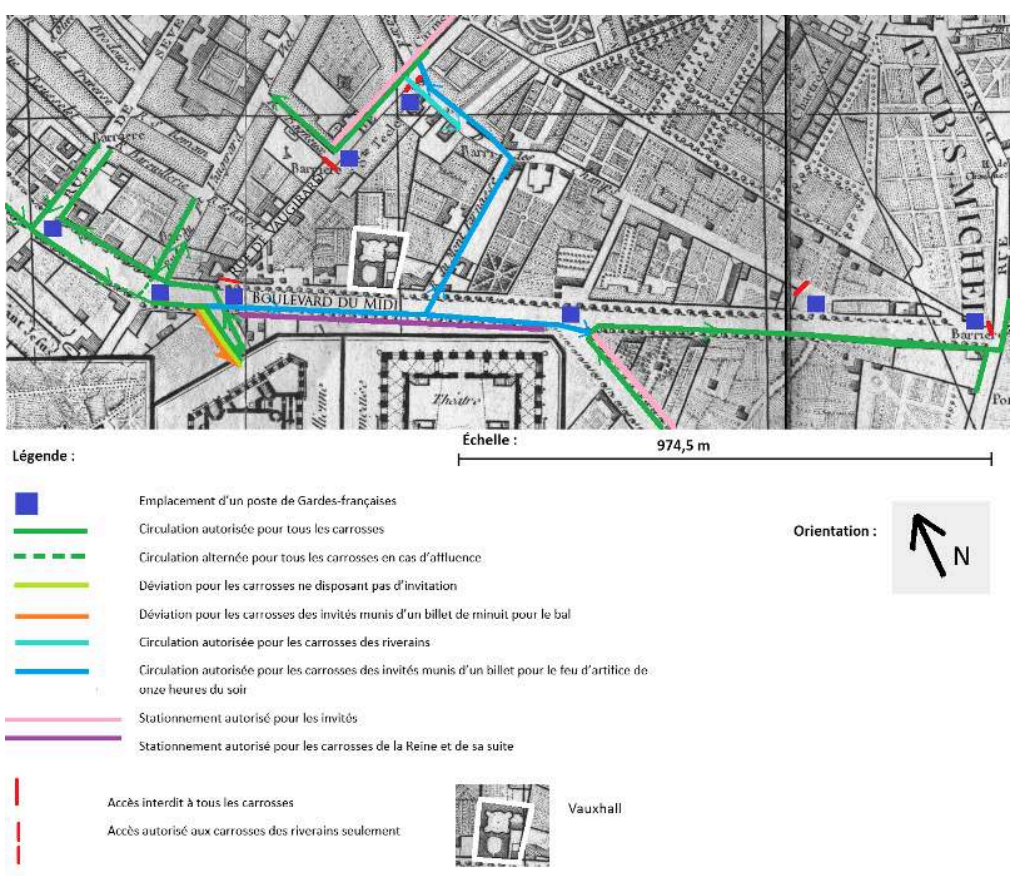

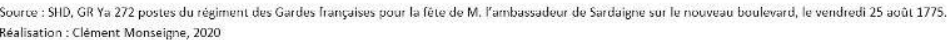

Fond de carte : Nouweau plon de Paris par Brion de la Tour, chez Campion fréres, Paris, 1787, numérisation par Pascal lerjan, CC By 2.0, via Wikimedia Commons.

Le tir d'un feu d'artifice attirait, en dehors des invités, une foule importante de curieux, dont il fallait assurer la circulation dans les rues, afin d'éviter toute bousculade en cas de mouvement de panique comme cela s'était produit le 30 mai 1770. Pour cela, il convenait en premier lieu d'éviter l'affluence des carrosses sur le boulevard du Midi. $\mathrm{Au}$-delà de son concours à la sûreté publique, cette mesure s'inscrivait dans une perspective plus large de recherche d'aménités à travers la régulation des circulations urbaines. Le XVIII siècle fut marqué par de nombreux travaux d'urbanisme pratique et utilitaire qui participèrent à «l'embellissement des villes » (Harouel, 1993). Dans cette perspective, le boulevard, à la fin de l'Ancien Régime, était envisagé comme un espace de promenade qui devait être épargné autant que possible par les embarras de la 
circulation des carrosses et il était en cela opposé au modèle de la rue (Turcot, 2005). Afin d'y parvenir, une portion du boulevard, entre la rue du Petit-Vaugirard et la barrière d'Enfer, fut mise en sens unique, une autre portion fut entièrement réservée à la circulation des carrosses des invités, qui pouvaient ensuite aller stationner dans la rue de Vaugirard ou sur la route de Sceaux pour y attendre leur maître; la portion du boulevard du Midi située devant le Vauxhall était quant à elle réservée au stationnement des carrosses de la Reine. La circulation était également régulée par le poste situé à l'intersection du boulevard et de la rue du Petit-Vaugirard, qui constituait le nœud problématique du plan de circulation puisque tous les flux y convergeaient. Ce poste avait donc pour consigne, en cas d'affluence de «faire entrer dans la file trois carrosses de la file qui vient du côté de la rue de Sève et trois de celle de la rue des vieilles Tuileries ${ }^{45} \aleph^{46}$. La rue de Vaugirard constituait, depuis l'intérieur de Paris, l'accès le plus proche et le plus important au Vauxhall. Les postes de Gardes-françaises furent donc chargés d'en réserver une portion à la circulation des piétons, comme une issue de secours au cas où surviendrait un mouvement de foule. Le tir du feu d'artifice faisait craindre, par ailleurs, une panique des chevaux auxquels les carrosses étaient attelés, puisque les postes devaient également « arrêter et ne laisser passer aucun carrosse au moment que les premières fusées partiront $»^{47}$.

Cette mise en ordre des mobilités par les soldats aux Gardes-françaises participait à un maintien de l'ordre public qui assurait la sécurité des Parisiens et qui révélait la capacité du pouvoir royal à ordonner l'espace. Comme le note Vincent Milliot, en analysant un dispositif similaire déployé à l'occasion de la naissance du Dauphin, en 1782 « les mesures de police "fabriquent" en quelque sorte de la "métropolité" » (2014 : 296). La participation du régiment au contrôle des circulations pouvait aussi revêtir une dimension politique plus évidente encore et révéler le lien étroit qui l'unissait à l'autorité royale.

\section{Réguler les mobilités pour maintenir l'ordre : l'exemple des troubles de la place Dauphine (27-31 août 1788)}

La décennie 1780 fut particulièrement agitée dans la généralité de Paris avec 101 actes rébellionnaires, recensés par l'Historical Social Conflict Database, contre 47 en moyenne pour les décennies précédentes du XviII siècle (Chambru, ManeuvrierHervieu, 2020). Mais la fin du XVIII ${ }^{\mathrm{e}}$ siècle vit également «l'entrée en politique des foules parisiennes » (Nicolas, $2002: 18$ ) lors des émeutes de la place Dauphine en août 1788. L'état désastreux des finances à l'été 1788 (Egret, 1962) précipita la disgrâce du ministre Loménie de Brienne, dont le départ provoqua des scènes de liesse dans Paris. Le 27 août, un rassemblement eut lieu sur la place Dauphine, l'effigie du ministre y fut brûlée et la foule brisa à coups de pierres les fenêtres des particuliers qui n'avaient pas illuminé leurs croisées à l'occasion de cet événement ${ }^{48}$. La foule parisienne exprimait ainsi une joie subversive et spontanée en se réappropriant les éléments emblématiques des réjouissances publiques étant donné que l'illumination des fenêtres était ordinairement imposée aux Parisiens par une ordonnance de police, lors des célébrations organisées dans la ville par la monarchie (Valade, 2019). Le lendemain, un conseil extraordinaire se réunit chez le maréchal de Biron. Il était composé du secrétaire d'État à la Maison du roi, Villedeuil, du lieutenant général de police, Thiroux de Crosne, du procureur du roi, des commandants du Guet et des Gardes-Suisses, Dubois et le comte d'Affry ainsi que de deux commissaires au Châtelet. Cette assemblée 
se concerta pour coordonner l'action des différentes forces du maintien de l'ordre, afin d'empêcher de nouveaux troubles ${ }^{49}$. Dès le soir, le régiment des Gardes-françaises et le Guet furent envoyés sur la place Dauphine, pour en filtrer l'accès ; seuls les riverains furent autorisés à entrer sur la place, pour cela « on laissait passer les gens vêtus décemment, mais tout ce qui avait l'air ouvrier était repoussé $~^{50}$. L'action des Gardesfrançaises était donc inspirée par une lecture du trouble à l'ordre public qui associait déjà les classes laborieuses aux classes dangereuses (Chevalier, 1958). Le dispositif de contrôle était ainsi fondé sur la perception de la condition sociale des individus à travers leur apparence (Roche, 1989) et leur lieu de résidence puisque les habitants de la place Dauphine étaient, à cette époque, particulièrement aisés (Ducoudray et al. 2000). Cette méfiance particulière des Gardes-françaises à l'égard des ouvriers parisiens s'inscrivait dans un contexte de multiplication des émotions ouvrières à la fin du XVIII ${ }^{\mathrm{e}}$ siècle (Nicolas, 2002). Au cours de ces événements, les soldats aux Gardes se bornèrent cependant à patrouiller dans Paris, et à protéger la place Dauphine, tandis que la Garde de Paris fut impliquée dans une confrontation violente avec la population.

Le 28 août 1788, au soir, des fantassins de la Garde de Paris, après avoir essuyé une pluie de pierres, avaient en effet asséné des coups de crosse et de baïonnette à la foule qui était massée sur le Pont-Neuf. Dans la mêlée, une cinquantaine de civils avaient été blessés ainsi que des hommes de la Garde de Paris qui comptait également des morts dans ses rangs selon le libraire Hardy ${ }^{51}$. Le lendemain, de nombreux particuliers allèrent se plaindre de la brutalité de la Garde de Paris auprès du Lieutenant civil du Châtelet, du Lieutenant général de police et des commissaires au Châtelet ${ }^{52}$. Cette affaire eut des suites judiciaires puisque le major du Guet fut cité à comparaitre devant le Parlement de Paris, le 24 septembre 1788, pour s'expliquer sur la conduite de ses hommes lors des événements d'août 1788. Les poursuites à son encontre furent cependant abandonnées en raison des pressions exercées sur le Parlement par Villedeuil, secrétaire d'État à la Maison du roi ${ }^{53}$. Les soldats de la Garde de Paris furent néanmoins la cible du ressentiment populaire ; le libraire Hardy observait en date du 29 août, au soir, « la jeunesse turbulente secondée de la populace qui avait projeté de venir leur déclarer une espèce de guerre ouverte $»^{54}$. Le 31 août, lorsque deux soldats de la Garde de Paris furent capturés, et la foule les obligea à demander pardon au pied de la statue d'Henri IV, sur le Pont-Neuf, pour avoir participé aux opérations de maintien de l'ordre qui furent menées brutalement par leur unité ${ }^{55}$. Cet épisode témoignait ainsi de la forte dimension symbolique des événements d'août 1788 à Paris et notamment de l'occupation de la place Dauphine.

\section{Contrôler les mobilités pour préserver les hiérarchies symboliques}

Cette place était en effet située entre la statue d'Henri IV et le Palais de la Cité qui abritait le Parlement de Paris. Ce dernier était entré dans les bonnes grâces de l'opinion parisienne en s'opposant à l'impôt sur le timbre de Loménie de Brienne en 1787, ce qui lui valut d'être exilé à Troyes au mois d'août (Figeac, 2006). La figure d'Henri IV, quant à elle, devint populaire au cours du XvIII ${ }^{\mathrm{e}}$ siècle (Chéry, 2010), ce dont témoignait parfaitement le comportement de la foule qui se rassembla autour de sa statue. Parmi les cris de joie qui s'élevèrent durant les événements d'août 1788, les observateurs purent ouïr « vive notre bon roi Henri IV, vive M. Necker ». Cette appropriation populaire de l'espace était profondément subversive, car elle contredisait l'usage symbolique que Louis XVI souhaitait lui aussi faire de la figure de son ancêtre. C'est en 
effet au cours du règne de Louis XVI que naquit le projet de reconvertir une partie de la place Dauphine en place Louis XVI, et de placer une statue équestre du monarque en regard de celle d'Henri IV (Deming, 1989). Les efforts déployés pour préserver la place Dauphine témoignent donc de considérations symboliques et politiques dans les stratégies du maintien de l'ordre. L'occupation de la place Dauphine était un enjeu symbolique pour le pouvoir royal comme pour la foule des émeutiers, ce qui peut expliquer les efforts qu'ils firent pour la reconquérir ; Hardy put observer le soir du 28 août : « la jeunesse secondée par une populace nombreuse, qui fait diverses tentatives pour forcer la Garde et s'emparer de la place Dauphine de différents côtés... $»^{56}$.

La place Dauphine faisait donc partie, aux yeux du pouvoir royal, des lieux qui constituaient " les "moments sacrés" d'un "plan" qu'ils contribuent à fonder, à définir c'est-à-dire à différencier... » (Raffestin, 2019 : 247). Les efforts du régiment des Gardesfrançaises pour préserver la sacralité de ce lieu de toute tentative de profanation peuvent être rapprochés des fonctions qu'il occupait auprès de la famille royale. Lors de la venue de la reine à Paris, le 21 janvier 1782, à l'occasion de ses relevailles, deux compagnies de Gardes-françaises furent requises pour délimiter un périmètre autour de l'abbaye Sainte-Geneviève, où devait se rendre la souveraine ${ }^{57}$. Les soldats furent postés aux principaux accès à l'abbaye et avaient pour ordre d'y filtrer les passages : « On laissera passer à pied, point en carrosse, les chevaliers de Saint-Louis. Aucun carrosse ne doit passer que ceux de la Reine, de la suite des Princes du Sang et de M. le gouverneur de Paris. Ces deux postes empêcheront la Garde de Paris d'entrer dans la rue Saint-Étienne-des-Grès, et dans le petit marché Saint-Jacques, il n’y a que le commandant de la Garde, le major et un ou deux cavaliers qui puissent entrer. $\wedge^{58}$

Le contrôle des circulations autour de l'abbaye Sainte-Geneviève dépassait donc les considérations pragmatiques relatives à la sécurité de la Reine, il s'agissait aussi de constituer un espace sacré (Eliade, 1965) autour d'une figure royale, un espace auquel même la Garde de Paris ne pouvait accéder. Ces préoccupations symboliques étaient encore visibles à travers l'ordre donné aux Gardes-françaises de ne se retirer qu'une demi-heure après le départ de la souveraine, montrant que la préservation de l'espace qui lui était réservé comptait tout autant que la préservation de sa personne ${ }^{59}$. La venue du roi ou de la reine à Paris, et leur apparition publique en présence des Gardesfrançaises contribuaient également à associer sur le plan symbolique les figures de la royauté à la puissance de l'armée, ainsi que l'explique Jacques-Louis Lantoine évoquant le concept de disposition chez Spinoza :

«Un corps affecté en même temps par deux corps à la fois - le corps du roi et le corps de l'armée par exemple - est disposé de telle sorte que, quand l'un ou l'autre se présente à nouveau, aussitôt la trace du second à laquelle est liée la trace du premier est éveillée, avec toute la charge affective que l'un des deux, par hypothèse, concentre : le visage du roi, indifférent, voire ridicule, évoque aussitôt la peur qu'avait fait naître l'armée. » $(2018: 37)$.

Cette proximité avec le pouvoir royal constituait la spécificité du régiment des Gardesfrançaises, qui, en maintenant l'ordre dans Paris, participait à l'affirmation de l'autorité royale sur la ville. 


\section{Les Gardes-françaises, acteurs d'une affirmation martiale de l'autorité royale sur Paris}

\section{Un régiment sous l'autorité exclusive du monarque}

En 1775, lors de la Guerre des Farines, le régiment des Gardes-françaises fut mobilisé pour contenir les émotions populaires qui secouaient Paris. Il fit preuve à cette occasion d'une grande modération. En effet, ses officiers étaient alors soumis au contrôle du Parlement de Paris qui disposait d'un droit de grande police lui permettant de citer en justice des officiers s'il estimait qu'une opération de maintien de l'ordre avait été menée trop brutalement (Chagniot, 1985). Les officiers aux Gardes refusèrent d'apporter un concours systématique au maintien de l'ordre en affirmant qu'ils craignaient la censure du Parlement. Malesherbes, secrétaire d'État de la Maison du roi, notait par exemple, en 1775, que lors de la Guerre des Farines, le régiment des Gardes avait laissé commettre des désordres sous ses yeux, car ses officiers « ne devaient ni de voulaient s'exposer à ce que le Parlement pût leur faire leur procès ${ }^{60}$. En réponse à ces arguments, le roi décida de dessaisir le Parlement de Paris de son droit de grande police afin que les officiers aux Gardes ne puissent plus invoquer la crainte d'un procès pour justifier leur inaction.

Dès lors, le régiment des Gardes-françaises était certes libéré du contrôle sur ses actions de police, néanmoins leur dissociation du Parlement rendait le roi seul responsable de la conduite du régiment. Ainsi, les Gardes-françaises et suisses étaient davantage liés à l'autorité royale que les autres forces de maintien de l'ordre, comme la Garde de Paris, qui demeuraient sous le contrôle du Parlement. Dans un pamphlet anonyme, publié en 1788 sous le titre Le parterre justifié, un particulier dénonçait en ces termes les actions du régiment des Gardes dans les théâtres « depuis longtemps, la police militaire et despotique du régiment des Gardes, révolte le public, d'où lui vient le privilège exclusif d'exercer une police sans être soumis à la justice civile ? ${ }^{61}$. À travers cette question rhétorique transparaissait une critique implicite de l'autorité royale que l'auteur prolongeait par la suite en invoquant le contre-pouvoir protecteur du Parlement et en prenant pour exemple le modèle politique anglais. Critiquer l'action du régiment des Gardes-françaises revenait à critiquer le roi puisque ces troupes étaient sous son autorité directe. Cette association devait être d'autant plus évidente pour les contemporains du pamphlet que le régiment était fortement lié à la figure royale sur le plan symbolique.

\section{«Ultima ratio regum ${ }^{62}$ » : le bras armé de l'autorité royale contre les élites contestataires}

Au printemps 1788, lorsque le Parlement de Paris se dressa contre la réforme de Lamoignon qui lui retirait notamment son droit d'enregistrement, le pouvoir royal procéda à une manifestation de force éclatante en envoyant le marquis d'Agout à la tête de six compagnies de Gardes-françaises dans l'enceinte du Palais de la Cité pour procéder à l'arrestation des conseillers Goislard de Montsabert et Duval d'Esprémesnil (Chagniot, 1985). Le Parlement de Paris fut ensuite occupé par des Gardes-françaises, dont la présence manifestait le contrôle de cet espace de contestation par le pouvoir royal. La participation du régiment à ce coup de force donna lieu à la publication de nombreux pamphlets satyriques qui tournaient en dérision la démesure du dispositif 
qui fut déployé : « qui pourra jamais croire que seul avec quinze cent hommes vous ayez pu vous rendre maitre d'un magistrat en robe et bonnet carré ? ${ }^{63}$ pouvait-on lire dans la Lettre de Desbrugnières, inspecteur de police à son confrère d'Agoult, capitaine aux Gardes-françaises. L'importance des effectifs envoyés au Parlement montre cependant, une nouvelle fois, que l'occupation de l'espace par les Gardes-françaises, au-delà des considérations pragmatiques, répondait aussi à des enjeux symboliques. Il s'agissait, en l'occurrence, de montrer que le roi pouvait compter sur une force armée fidèle et considérable pour rétablir l'ordre politique, et surtout qu'il ne craignait point de le faire avec fracas et ostentation, en dépit du soutien populaire dont bénéficiaient les magistrats.

La participation au maintien de l'ordre dans Paris, d'une unité militaire étroitement liée au pouvoir royal, était capitale pour ce dernier. Il s'agissait en effet de conjurer l'apparition d'un "Anti Versailles $~^{64}$ dans une ville qui, à l'issue d'un processus de centralisation, était devenue à la fin du XVIII ${ }^{e}$ siècle, un enjeu politique majeur dont dépendait le reste du royaume. Ce qui devait inquiéter le parti du roi était davantage le soutien de l'armée à un contre-pouvoir parisien que le soutien d'une population désarmée (Chagniot, 1985), d'où l'absolue nécessité de maintenir à demeure dans la ville une unité de la Maison militaire du roi, dont la fidélité à la monarchie semblait être assurée par son lien étroit avec elle et par les nombreux égards dont elle était honorée. Il est ainsi possible d'affirmer que la présence des Gardes-françaises à Paris n'était pas seulement destinée à contenir les foules turbulentes, mais qu'elle représentait également un message adressé aux élites contestataires.

\section{L'efficacité d'une unité militaire face aux foules}

Le monopole royal de la force armée à Paris était quotidiennement rappelé aux habitants de la ville par la présence de Gardes-françaises en armes dans les lieux publics qui jouaient ainsi un rôle dissuasif. La mise en scène de l'armement dont disposaient les soldats des Gardes-françaises (La Sabretache, 1987) était un spectacle que pouvaient parfois observer les passants dans l'espace public comme le remarquait Louis-Sébastien Mercier :

«Ces soldats qui accompagnent les productions de Racine et celles de M. Piis-Barré, font à quatre heures des évolutions militaires sur la place, comme s'ils allaient à l'ennemi. On les voit distinctement mettre la balle dans le fusil : voilà le prélude de la comédie. Cela n'est pas trop gai avant une représentation du Bourgeois gentilhomme $"^{65}$

Cette scène est tout à fait intéressante pour comprendre comment une unité qui déployait quotidiennement un millier d'hommes seulement pouvait concourir efficacement au maintien de l'ordre dans une ville qui comptait environ 600000 habitants (Ducoudray et al. 2000). Lorsqu'ils devaient maintenir le bon ordre face à une foule nombreuse, les soldats pouvaient compenser leur infériorité numérique par une occupation martiale de l'espace, il s'agissait de montrer que les forces militaires, avantagées par leur armement, étaient en mesure de tenir un territoire donné. De telles manœuvres contribuaient à forger, au sein de la population, un imaginaire autour des unités militaires du maintien de l'ordre, qui pouvaient inspirer la crainte par leur seule présence face à une foule. Une gazette à la main conservée aux Archives nationales notait à propos des troubles de la place Dauphine que si les Gardes-françaises s'étaient mêlés au conflit qui opposait la foule aux soldats de la Garde de Paris « il y aurait eu sans doute bien du sang répandu $»^{66}$. En revanche, la population parisienne se moquait 
sans crainte des soldats de cette dernière unité, qui avaient acquis, par exemple, le surnom de « lapins ferrés » (Chagniot, 1973).

Le fusil dont disposaient les Gardes-françaises les contraignait à adopter une tactique sédentaire du maintien de l'ordre, qui s'avérait assez efficace lorsqu'ils devaient tenir un espace face à la foule. Le 28 avril 1789, des ouvriers parisiens, que le duc du Châtelet avait réussi à apaiser la veille, projetèrent à nouveau de s'en prendre à la manufacture de Jean-Baptiste Réveillon. Afin de la protéger, une cinquantaine de grenadiers des Gardes-françaises, avec un sergent à leur tête, furent envoyés dans la rue de Montreuil (Collot, 1934). Les cinquante Gardes-françaises firent face à une foule considérable qu'ils parvinrent à tenir à l'écart de la manufacture en érigeant des barricades dans la rue de Montreuil. Si la barricade est fortement associée à l'insurrection et à la révolte (Corbin, Mayeur, 1997 ; Traugott, 2010), elle était également un élément important de la stratégie militaire à l'époque moderne ${ }^{67}$. Appliquée au maintien de l'ordre, elle s'avérait un outil efficace puisque les Gardes-françaises réussirent à tenir le terrain jusqu'au passage de la voiture de la duchesse d'Orléans qui les obligea à ouvrir une brèche dans la barricade, dans laquelle s'engouffrèrent les émeutiers. Le sergent, Jean-Nicolas Thiébault, qui commandait le détachement ordonna alors à ses hommes de se retirer ${ }^{68}$. Il fut pour cela congédié du régiment par ordre du duc du Châtelet ${ }^{69}$. Les Gardesfrançaises qui furent appelés en renfort demeurèrent en retrait tandis que les cavaliers du régiment Royal-Cravatte, qui constituaient les éléments mobiles du dispositif de maintien de l'ordre, tentaient de disperser la foule. Les fantassins entrèrent ensuite en action, ils tirèrent une première décharge à blanc pour intimider les émeutiers qui s'engouffrèrent immédiatement dans les maisons, d'où ils jetèrent des pierres et des tuiles sur la troupe, qui reçut alors l'ordre de tirer à balles réelles (Collot, 1934). La participation des Gardes-françaises à cette opération de maintien de l'ordre couvrit les soldats d'opprobre ; le 2 mai, une femme s'en alla « clabauder et dire des sottises atroces $»^{70}$ aux soldats qui étaient en faction devant la caserne de la rue Mouffetard (Burstin, 2005).

Cette nouvelle macule dans l'opinion parisienne, qui s'ajoutait à la participation des Gardes-françaises à la répression de la contestation parlementaire, suscita une grave crise morale dans le régiment. Les soldats aux Gardes-françaises qui furent quotidiennement exposés à la réprobation de leur action par les Parisiens finirent par refuser de prêter main-forte à l'autorité royale. Le 23 juin 1789, les Gardes-françaises mirent l'arme au pied lorsqu'on leur ordonna de réprimer une foule pacifique qui se présentait au château de Versailles ${ }^{71}$. "C'est que, dans un État moderne, il ne suffit plus d'avoir le contrôle de la force publique pour rétablir l'ordre, il faut encore conserver ou retrouver l'adhésion du peuple, sans quoi l'armée elle-même finit par se récuser » (Chagniot, $1985: 28$ ).

\section{Conclusion}

5 L'étude du rapport du régiment des Gardes-françaises à l'espace parisien à la fin de l'Ancien Régime a permis de mettre en lumière les différentes dimensions revêtues par l'action de cette unité. La surveillance des rues de Paris, assurée quotidiennement par le régiment des Gardes, contribuait à la fois à la sûreté de la ville et au contrôle des foyers d'agitation populaire. Elle était ponctuée par des opérations à caractère politique qui permettaient au pouvoir royal de réaffirmer son autorité, face aux foules et aux élites contestataires, en investissant symboliquement les lieux de pouvoir de 
l'espace parisien. Le lien étroit entretenu entre les Gardes-françaises et le roi faisait de ces soldats des relais puissants de l'autorité du monarque. En occupant l'espace et en contrôlant les mobilités, les Gardes-françaises permettaient à l'autorité royale d'affirmer sa présence dans une ville qui avait été désertée par le souverain un siècle plus tôt, mais qui demeurait un centre de pouvoir majeur. Le contrôle symbolique des lieux de pouvoir dans l'espace parisien était assuré de manière ostentatoire par les Gardes lors de missions ponctuelles, mais c'est la présence quotidienne des soldats dans Paris qui contribuait à inscrire ce contrôle de l'espace dans le temps. C'est précisément parce que la sûreté publique et le maintien de l'ordre étaient assurés par une même unité, proche du pouvoir royal, qu'il est possible de parler de maintien de l'ordre public, social et politique. Ces fonctions dissuasives et répressives du régiment coexistaient également avec différentes missions d'intérêt public comme la lutte contre les incendies (Chagniot, 1985), et c'est cette confusion qui permettait aux Gardesfrançaises de légitimer leur action auprès des Parisiens.

Les soldats du régiment des Gardes et leurs bas-officiers furent happés au cours du mois de juillet 1789 par le nouveau pouvoir municipal, qui les employa à affirmer son autorité naissante. Les anciens soldats intégrèrent la nouvelle Garde nationale soldée et participèrent ainsi à l'avènement d'un nouveau pouvoir national en assurant le maintien de l'ordre (Carrot, 1995), comme ils l'avaient fait sous l'Ancien Régime, ainsi que l'affirmait le député Jacques Menou en 1791 :

«Paris, lieu des séances de l'Assemblée nationale, est le centre du mouvement de toutes les parties de la monarchie [...] Il est donc de toute vérité, Messieurs, que c'est à la tranquillité et à l'ordre public maintenu dans Paris que la France entière doit et la Révolution et la constitution. $»^{72}$

7 Mais c'est également au cours de la période révolutionnaire qu'émergea une réflexion sur les prérogatives des différentes composantes de la force publique. Le comte de Guibert, dans son ouvrage De la force publique ${ }^{73}$ proposait une distinction fondamentale entre les forces du dehors, destinées à la guerre et les forces du dedans, destinées au maintien de l'ordre (Drévillon, 2018 ; Milliot et al., 2020). Cette conception fut adoptée par les législateurs qui mirent un terme à la confusion entre les différentes fonctions des forces armées au service de l'État. Les anciens soldats aux Gardes-françaises, exemples éloquents du mélange des prérogatives, furent finalement exclus de la Garde nationale soldée et incorporés à l'armée de ligne et à la gendarmerie par la loi du 28 août $1791^{74}$.

\section{BIBLIOGRAPHIE}

ABAD R. (2007), « La fraude dans le commerce et l'approvisionnement alimentaires de Paris au XVIIIe siècle. Aperçu d'ensemble et étude du cas des vins frelatés ", in Béaur G., Fraude, contrefaçon, contrebande de l'Antiquité à nos jours, Genève, Droz, pp. 539-561.

ALESSI G. (2002), « La comparsa di una polizia “moderna” », in Antonielli L., La polizia in Italia nell'età moderna, Rubbettino, Soveria Manelli, pp. 33-39. 
BLANC-CHALÉARD M.-C., DOUKI C., DYONET N., MILLIOT V. (2001), Police et migrants, Rennes, Presses universitaires de Rennes.

BROUILLET P. (1998), «L'organisation de la maréchaussée dans la généralité de Paris à la fin de l'Ancien Régime », Revue historique des Armées, no 4, pp. 3-14.

BROUILLET P. (2002), « Armée et maintien de l'ordre en France dans la seconde moitié du XVIIIe siècle (1750-1789) », in Armée et maintien de l'ordre, cahier du Centre d'études de la défense, Paris, ministère de la Défense.

BROUILLET P. (2008), « La compagnie de maréchaussée de l'île-de-France et la Garde de Paris : deux unités de police ; un même métier ? ", in Berlière J.-M., Denys C., Kalifa D. et Milliot V., Métiers de police, Rennes, Presses universitaires de Rennes, pp. 77-86.

BURSTIN H. (2005), Une révolution à l'œuvre : le faubourg Saint-Marcel (1789-1794), Seyssel, Champ Vallon.

CARROT G. (1995), Révolution et maintien de l'ordre, 1789-1799, Paris, SPM.

CHAMBRU C., MANEUVRIER-HERVIEU P. (2020), Historical Social Conflict Database, consulté le 20 novembre 2020.

URL : https://www.unicaen.fr/hiscod/

CHEVALIER L. (1958), Classes laborieuses et classes dangereuses à Paris pendant la première moitié du XIXe siècle, Paris, Plon.

CHAGNIOT J. (1973), « Le Guet et la Garde de Paris à la fin de l'Ancien Régime », Revue d'histoire moderne et contemporaine, tome 20 , no 1 , pp. 58-71.

CHAGNIOT J. (1974), « Le problème du maintien de l'ordre à Paris au XVIIIe siècle », Bulletin de la Société d'histoire moderne et contemporaine, 15e série, no. 8, pp. 32-45.

CHAGNIOT J. (1977), « Une panique : les Gardes Françaises à Dettingen (27 juin 1743) », Revue d'histoire moderne et contemporaine, tome 24, no. 1, pp. 78-95.

CHAGNIOT J. (1985), Paris et l'armée au XVIIIe siècle, Paris, Économica.

CHAGNIOT J. (1988), Nouvelle histoire de Paris. Paris au XVIIIe siècle, Paris, Hachette.

CHÉRY A. (2010), « Louis XVI ou le nouvel Henri IV », Bulletin du Centre de recherche du château de Versailles, [En ligne]

URL : http://journals.openedition.org/crcv/1046

CICCHINI M. (2009), « La République transparente ? Un projet de quadrillage policier à Genève autour de 1779 », in Denys C., Marin B. et Milliot V., Réformer la police : les mémoires policiers en Europe au XVIIIe siècle, Rennes, Presses universitaires de Rennes, pp. 21-45.

COLLOT J. (1934), « L'affaire Réveillon », Revue des questions historiques, tome 121, pp. 35-55.

CONCHON A. (2018), « Policer les circulations en France au XVIIIe siècle », in : Conchon A., Montel L., Regnard C., Policer les mobilités, Paris, Éditions de la Sorbonne, pp. 15-30.

CORBIN A., MAYEUR J.-M. (1997), La barricade, Paris, Éditions de la Sorbonne.

CORVISIER A., DELMAS J. (1992), Histoire militaire de la France. 2. De 1715 à 1872, Paris, Presses universitaires de France.

DEMING M. K. (1989), « Louis XVI en l'île. Contribution à l'étude des places royales parisiennes à la fin de l'Ancien Régime. ", Revue de l'Art, no 83, pp. 86-92.

DOI : https://doi.org/10.3406/rvart.1989.347763 
DENYS C. (2003), « Logiques territoriales : la territorialisation policière dans les villes au XVIIIe siècle ", Revue d'histoire moderne et contemporaine, tome 50, no 1, pp. 13-26.

DENYS C. (2008), « Institutions, corps, services », in Berlière J.-M., Denys C., Kalifa D. et Milliot V., Métiers de police, Rennes, Presses universitaires de Rennes, pp. 37-43.

DRÉVILLON H. (2018), Histoire militaire de la France, Paris, Perrin, 2018, 864 p.

DUCOUDRAY É., MONNIER R., ROCHE D., LACLAU A. (2000), Atlas de la Révolution française. 11. Paris, Paris, Éditions de l'École des hautes études en sciences sociales.

EGRET J. (1962), La pré-révolution française : (1787-1788), Paris, Presses universitaires de France.

ELIADE M. (1965), Le sacré et le profane, Paris, Gallimard.

FARGE A. (1979), « Les théâtres de la violence à Paris au XVIIIe siècle », Annales : Économies, sociétés, civilisations, 34e année, no 5, pp. 984-1015.

DOI : https://doi.org/10.3406/ahess.1979.294104

FIGEAC M. (2006), « Les magistrats en révolte en 1789 ou la fin du rêve politique de la monarchie des juges ", Histoire, économie \& société, vol. 25, no. 3, pp. 385-400

DOI : https://doi.org/10.3406/hes.2006.2608

GERMES M., 2014, « Cartographies policières : la dimension vernaculaire du contrôle territorial », EchoGéo, no. 28, [En ligne].

DOI : https://doi.org/10.4000/echogeo.13856

GOMEZ PARDO J. (2012), La maréchaussée et le crime en Île de France : sous Louis XIV et Louis XV, Paris, Les Indes savantes.

HAROUEL J.-L. (1993), L'embellissement des villes. L'urbanisme français au XVIIIe siècle, Paris, Picard.

HERBERT S. (1996), «The geopolitics of the police: Foucault, disciplinary power and the tactics of the Los Angeles Police Department », Political Geography, vol. 15, no. 1, pp. 47-57.

DOI : https://doi.org/10.1016/0962-6298(95)00004-6

KARÉIEV N.-I. (1912), La densité de population des différentes sections de Paris pendant la Révolution, Paris, Honoré Champion.

LANTOINE J.-L. (2018), Spinoza après Bourdieu : politique des dispositions, Paris, Éditions de la Sorbonne, $138 \mathrm{p}$.

LA SABRETACHE (1987), « Le régiment des Gardes-françaises », Carnet de la Sabretache no. 90, pp. 133-164.

LEPETIT B. (1988), Les villes dans la France moderne, 1740-1840, Paris, Albin Michel.

MARIN B. (1993), « Découpage de l'espace et contrôle du territoire urbain : les quartiers de police à Naples (1779-1815) », Mélanges de l'École française de Rome, pp. 349-374

DOI : https://doi.org/10.3406/mefr.1993.4281

MILLIOT V. (2003), « Saisir l'espace urbain : mobilité des commissaires et contrôle des quartiers de police à Paris au XVIIIe siècle ", Revue d'histoire moderne et contemporaine, tome 50, no. 1, pp. $54-80$

DOI : https://doi.org/10.3917/rhmc.501.0054

MILLIOT V. (2014), " "Divise et commande" ou le rêve de Guillauté. Essai sur les pratiques policières de l'espace à Paris au XVIIIe siècle ", in Bergel P. et Milliot V., La ville en ébullition : Sociétés urbaines à l'épreuve, Rennes, Presses universitaires de Rennes, pp. 269-305. DOI : https://doi.org/10.4000/books.pur.50258. 
MILLIOT V. (2016), L'admirable police : tenir Paris au siècle des Lumières, Ceyzérieu, Champ Vallon.

MILLIOT V., BLANCHARD E., DENIS V., HOUTE A. (2020), Histoire des polices en France, des guerres de Religion à nos jours, Paris, Belin.

NICOLAS J. (2002), La rébellion française : mouvements populaires et conscience sociale : (1661-1789), Paris, Éditions du Seuil.

OGBORN M. (1993), « Ordering the City : surveillance, public space and the reform of urban policing in England, 1835-1856, Political Geography, no. 12, pp. 505-521.

PORTET P. (2012), « La mesure de Paris », in Charbonnier P., Les anciennes mesures du Centre historique de la France d'après les tables de conversion, Paris, Éditions du CTHS, pp. 13-54.

URL : https://hal.archives-ouvertes.fr/halshs-01672844v1

RAFFESTIN C. (2019), Pour une géographie du pouvoir, Lyon, ENS Éditions, 344 p. DOI : https://doi.org/10.4000/books.enseditions.7644.

RITTMANN A., JUINIÉ J. (1906), Atlas de la censive de l'archevêché dans Paris, Paris, Imprimerie nationale.

RAVEL J. S. (1999), The contested parterre : public theatre and French political culture, 1680-1791, Londres, Cornell University Press.

ROCHE D. (1989), La culture des apparences : une histoire du vêtement (XVIIe-XVIIIe siècle), Paris, Fayard.

ROCHE D. (2000), La ville promise : mobilité et accueil à Paris (fin XVIIe - début XIXe siècle), Paris, Fayard.

ROMON C. (1982), « Le monde des pauvres à Paris au XVIIIe siècle », Annales. Économies, Sociétés, Civilisations, 37e année, no. 4, pp. 729-763

DOI : https://doi.org/10.3406/ahess.1982.282884

SHERMAN L. W. et WEISBURD D. (1995), «General deterrent effects of police patrol in crime "hot spots" : a randomized, controlled trial », Justice Quarterly, no. 12, pp. 625-648.

TOURNIER M. (2004), «Émotion populaire, petite note lexicologique », Mots. Les langages du politique, no. 75, pp. 121-125.

TRAUGOTT M. (2010), The insurgent barricade, Los Angeles, University of California Press.

TURCOT L. (2005), « L 'émergence d'un espace plurifonctionnel : les boulevards parisiens au XVIIIe siècle », Histoire urbaine, vol. 1, no. 12, pp. 89-115.

DOI : https://doi.org/10.3917/rhu.012.0089

VIDONI N. (2015), « La lieutenance générale de police et l'espace urbain parisien. Pratiques, savoirs et contrôle du territoire (1667-1789) », Società e storia, pp. 459-486.

VIDONI N. (2018), « Le voisinage comme catégorie policière à Paris et Montpellier au XVIIIe siècle ", in Besse L., Cogné A., Krampl U. et Sauget S., Voisiner. Mutations urbaines et construction de la cité du Moyen Âge à nos jours, Tours, Presses universitaires François Rabelais, pp. 101-117.

WILLIAMS S. et COUPE T. (2017), « Frequency vs. Lenght of hot spots patrols : a randomised controlled trial », Cambridge Journal of Evidence-Based Policing, vol. 1, pp. 5-21.

DOI : https://doi.org/10.1007/s41887-017-0003-1 
VALADE P. (2019), « La couleur des réjouissances monarchiques », Dix-huitième siècle, vol. 1, no. 51, pp. 37-38.

DOI : https://doi.org/10.3917/dhs.051.0031

\section{Catalogue des sources}

\section{Fonds du Service historique de la Défense}

Sous série Ya, archives administratives du département de la Guerre :

Ya 269 Gardes françaises : ordonnances et mémoires, composition du régiment le 29 janvier 1764, réorganisation de 1777, réforme de 1789.

Ya 271 Gardes-françaises : petit état-major, état de soldats tailleurs (1771-1789) ; bas-officiers ; musique : mémoires, lettres, états (1777-1791) ; élèves du dépôt (1771-1787) ; troupe, récompense militaire et Invalides (1764-1789) ; modèles attachés à l'Académie royale de peinture (1776-1777).

Ya 272 Gardes-françaises : service, campagnes, police des rues, théâtres, garde auprès du roi ; troubles, fêtes publiques, funérailles à Saint-Denis, campagnes, cérémonies, chasse, incendies.

Ya 274 Gardes-françaises : affaires de discipline, délits de particuliers, discipline du corps, états de soldats punis (1755-1789) ; crimes et délits commis par des Gardes-françaises (1684-1770).

Ya 275 Gardes-françaises : armes, équipements (1753-1789), vivres (1741-1789), boulangerie (1774-1789), logement, casernement (1650-1789), hôpitaux (1705-1781) ; casernes, baux de location (1760-1789).

\section{Fonds de la BnF}

LA CHESNAYE DES BOIS F.-A.-A. (1758), Dictionnaire militaire portatif contenant tous les termes propres à la guerre, tome premier, Paris, chez Duchesne, $659 \mathrm{p}$.

E.M.L (1788), Le parterre justifié ou précis historique, Londres.

État du régiment des Gardes-françaises par rang de compagnie, éditions annuelles de 1744 à 1789 , Paris, Imprimeries de Lamesle et de Thiboust.

GUIBERT J.-A.-H. de (1790), De la force publique considérée dans tous ses rapports, Paris, Didot, $1790,196 \mathrm{p}$.

HARDY S.-P., (1787-88), Mes loisirs ou journal d'événements tels qu'ils parviennent à ma connaissance, volume VII, $484 \mathrm{p}$.

HARDY S.-P., (1788-89), Mes loisirs ou journal d'événements tels qu'ils parviennent à ma connaissance, volume VIII, $514 \mathrm{p}$.

Loi du 28 août 1791, relative à la nouvelle organisation de la Garde nationale soldée.

MERCIER L.-S., (1783), Tableau de Paris, tome premier, édition d'Amsterdam, 188 p.

MERCIER L.-S., (1783), Tableau de Paris, tome sixième, édition d'Amsterdam, 328 p.

MONIN H. (1898), L'État de Paris en 1789 : études et documents sur l'Ancien Régime à Paris, Paris, D. Jouaust, $688 \mathrm{p}$.

Ordonnance du Roi, concernant le régiment des Gardes-françaises de Sa Majesté, du 29 janvier 1764. 
Ordonnance du Roi, concernant le régiment des Gardes-françaises de Sa Majesté, du 17 juillet 1777.

Plan de travail pour la sûreté de Paris, Nouvelles acquisitions françaises, Ms. 3247, folio $132 \mathrm{v}^{\circ}$.

TARDIEU DE MALEISSYE A.-C. (1887), Mémoires d'un officier aux Gardes-françaises, Paris, E. Plon, $395 \mathrm{p}$.

\section{Fonds des Archives nationales}

K//160 Pièces manuscrites et imprimées relatives à l'agitation lors des lits de justice de 1787 et 1788 .

K//526 Acquisition des biens, effets et bâtiments des Gardes-françaises en août1789.

\section{NOTES}

1. Service historique de la Défense [désormais SHD], GR Ya 269, extrait de la revue faite le 30 avril 1789 à Paris et à Versailles aux six bataillons du régiment des Gardes-françaises.

2. Le régiment comptait trente compagnies, dont six compagnies de grenadiers, réparties en six bataillons. Chaque compagnie de Gardes-françaises était composée d'une centaine de soldats, commandés par des bas-officiers : huit caporaux et six sergents dont un premier sergent et un sergent-major. À la tête des compagnies se trouvait un capitaine qui commandait à des officiers subalternes: un capitaine en second dans les compagnies de grenadiers, deux lieutenants, deux sous-lieutenants, et deux enseignes dans les compagnies de fusiliers.

3. SHD, GR Ya 272.

4. Les Gardes-suisses remplissaient des fonctions similaires aux Gardes-françaises, ils disposaient de postes fixes dans Paris, mais ils étaient principalement casernés à Rueil et Courbevoie d'où ils pouvaient intervenir exceptionnellement lors de troubles. La milice provinciale pouvait elle aussi fournir un renfort extraordinaire, mais elle ne remplissait aucune fonction régulière dans le maintien de l'ordre. Les Invalides, enfin étaient chargés de la garde de lieux qui n'avaient plus d'importance stratégique à la fin du XVIII e siècle : la Bastille ou encore le Louvre.

5. On entend par unités de police active, les forces qui se consacraient exclusivement aux missions de sûreté et de maintien de l'ordre.

6. La Garde de Paris était, avec les Gardes-françaises, la principale force publique qui assurait un service quotidien dans le maintien de l'ordre à Paris, en effectuant des patrouilles et en intervenant lors des troubles. Les autres unités avaient des fonctions plus spécifiques. Les Gardes de la Ville étaient attachés au service de l'Hôtel de Ville. La compagnie de la Robe Courte, attachée aux cours de justice, était la seule unité habilitée à pénétrer en armes dans les églises, et à la fin du XVIII ${ }^{e}$ siècle, elle intervenait essentiellement dans ces cas précis. La compagnie de la Connétablie, quant à elle, devait notamment traiter les affaires des duels, en surveillant par exemple les assignations à résidence de nobles duellistes. La prévôté de l'île enfin, était rattachée à la maréchaussée, elle avait en charge la garde des postes d'octroi et la surveillance de la banlieue de Paris.

7. Terme générique désignant à la fin du XVIII ${ }^{\mathrm{e}}$ siècle les divers débits de boisson.

8. SHD, GR Ya 275, Casernement des Gardes-françaises, nouveau mémoire donné au Roi, le 30 mai 1774.

9. SHD, GR Ya 275, état des casernes du régiment des Gardes françaises suivant la visite faite par M. le Major. 
10. Archives nationales [désormais $\mathrm{AN}], \mathrm{K} / / 526$, Mémoire présenté à messieurs les représentants des Communes de Paris, par les propriétaires des casernes construites pour le régiment des Gardes-françaises.

11. État du régiment des Gardes-françaises par rang de compagnie, Paris, Imprimerie de Lamesle, édition annuelle de 1744 à 1789 , exemplaire consulté : année 1788.

12. Le grade d'enseigne était le premier échelon de la hiérarchie des officiers, dans les compagnies de fusiliers du régiment des Gardes-françaises.

13. La deuxième compagnie du deuxième bataillon occupait par exemple un rang supérieur à la deuxième compagnie du troisième bataillon qui avait elle-même la préséance sur la troisième compagnie du deuxième bataillon, etc.

14. Ordonnance du roi concernant le régiment des Gardes-françaises de Sa Majesté, du 29 janvier 1764, article III.

15. SHD GR Ya 272, ordre du 17 juin 1789.

16. État du régiment des Gardes-françaises par rang de compagnie, Paris, Imprimerie de Lamesle, 1789, p. 42.

17. État du régiment des Gardes-françaises par rang de compagnie, Paris, Imprimeries de Lamesle et de Thiboust, exemplaires consultés : de 1780 à 1788 .

18. SHD GR Ya 269, extrait de la revue faite le 30 avril 1789 à Paris et à Versailles aux six bataillons du régiment des Gardes-françaises ; extrait de la revue faite en juillet 1789 pour servir au paiement des appointements.

19. État du régiment des Gardes-françaises par rang de compagnie, Paris, Imprimerie de Lamesle, édition annuelle de 1744 à 1789, exemplaire consulté : année 1788.

20. Les compagnies du régiment des Gardes-françaises étaient baptisées suivant le patronyme des capitaines qui les commandaient.

21. BnF, État du régiment des Gardes-françaises... op. cit.

22. Les émotions populaires désignaient à la fin du XVIII ${ }^{\mathrm{e}}$ siècle les émeutes et les révoltes qui troublaient l'ordre public. Cette expression soulignait le lien étroit que l'action collective pouvait entretenir avec les affects (Tournier, 2004).

23. Bibliothèque nationale de France [désormais BnF], S.-P. Hardy, Mes loisirs ou journal d'événements tels qu'ils parviennent à ma connaissance, volume VIII, 1788-1789, p. 297.

24. SHD, GR Ya 272, garde de l'opéra du Palais-Royal.

25. Ordonnance du Roi, concernant le régiment des Gardes-françaises de Sa Majesté, du 17 juillet 1777, titre IV.

26. SHD, GR Ya 272, lettre du marquis du Sauzay au maréchal de Biron, sans date (elle s'inscrit dans l'échange épistolaire entre Calonne et Biron en décembre 1785).

27. La retraite désigne le moment où les soldats d'un régiment doivent regagner leur caserne.

28. SHD, GR Ya 272, lettre du marquis du Sauzay au maréchal de Biron, sans date.

29. SHD, GR Ya 274, état des soldats qui ont été punis depuis le samedi 9 mai 1789.

30. BnF, Plan de travail pour la sûreté de Paris, NAF 3247, folio $132 \mathrm{v}^{\circ}$.

31. La bataille de Dettingen en 1743 , au cours de laquelle des soldats du régiment des Gardesfrançaises se jetèrent dans le Main lors d'une retraite chaotique, fut à l'origine de nombreuses railleries dans lesquelles les soldats étaient comparés à diverses espèces de canards ou des poissons (Chagniot, 1977).

32. BnF, S.-P. Hardy, Mes loisirs ou journal d'événements tels qu'ils parviennent à ma connaissance, volume VII, 1787-1788, p. 189.

33. SHD, GR Ya 272, ordre renouvelé par M. le Major en date du $1^{\mathrm{er}}$ janvier 1788 concernant les patrouilles des caporaux pendant le cours de l'année.

34. L'itinéraire de la patrouille du poste de la rue de la Jussienne n'a pas été retracé étant donné qu'il était indiqué sur l'ordre du $1^{\mathrm{er}}$ janvier 1788 concernant ces patrouilles que : « ce poste ne fait point de patrouille de l'ordre de M. le Major jusqu'à nouvel ordre. » (SHD GR Ya 272). 
35. SHD, GR Ya 271, état des élèves du dépôt au 20 octobre 1782.

36. L.-S. Mercier, Tableau de Paris, tome 1, édition d'Amsterdam, 1783, p. 157.

37. SHD, GR Ya 272, ordre renouvelé par M. le Major en date du $1^{\mathrm{er}}$ janvier 1788 concernent les patrouilles des caporaux au cours de l'année.

38. Le calcul des distances parcourues par les patrouilles a été effectué en prenant pour points de départ et d'arrivée les casernes et en se basant sur le tracé des voies de circulation et non sur celui des itinéraires qui s'en écarte parfois afin de préserver la nomenclature des rues sur le plan de Brion de la Tour.

39. SHD, GR Ya 272, ordre renouvelé par M. le Major en date du $1^{\mathrm{er}}$ janvier 1788 concernent les patrouilles des caporaux au cours de l'année.

40. Citation en langue originale : Disciplinamento materiale del territorio.

41. L.-S. Mercier, Tableau de Paris, op. cit., p. 68.

42. L.-P. de Ségur, Mémoires ou souvenirs et anecdotes, tome premier, Turin, chez les frères Reycend, 1829, p. 41.

43. Les Vauxhalls étaient des bâtiments d'origine britannique, qui abritaient, à la fin du $\mathrm{XVIII}^{\mathrm{e}}$ siècle, des activités de divertissement comme des fêtes ou des bals.

44. SHD, GR Ya 272, postes du régiment des Gardes françaises pour la fête de M. l'ambassadeur de Sardaigne sur le nouveau boulevard, le vendredi 25 août 1775.

45. Nom alternatif de la rue du Petit-Vaugirard.

46. SHD, GR Ya 272, postes du régiment des Gardes françaises pour la fête de M. l'ambassadeur de Sardaigne sur le nouveau boulevard, le vendredi 25 août 1775 .

47. SHD, GR Ya 272, ibid.

48. $\mathrm{AN}, \mathrm{K} / / 160$ : pièces manuscrites et imprimées relatives à l'agitation lors des lits de justice de 1787 et 1788 , pièce $n^{\circ} 6,64$, gazette à la main, non signée, « Paris au 29 août ».

49. «Exposé lu au Parlement par le major du Guet, de ce qui s'est passé à Paris depuis qu'on y a reçu la nouvelle de la nomination de $\mathrm{M}$. Necker au ministère ", in : H. Monin, L'État de Paris en 1789 : études et documents sur l'Ancien Régime à Paris, Paris, D. Jouaust, 1889, p. 483.

50. AN, K//160 : pièces manuscrites et imprimées relatives à l'agitation lors des lits de justice de 1787 et 1788 , pièce $n^{\circ} 6,64$, gazette à la main, non signée, «Paris au 29 août ».

51. BnF, S.-P. Hardy, Mes loisirs..., volume VIII, op. cit., p. 61.

52. BnF, S.-P. Hardy, ibid.

53. «Extraits de la séance plénière du Parlement du 24 septembre 1788 », in : H. Monin, L'État de Paris..., op. cit., pp. 482-487.

54. BnF, S.-P. Hardy, Mes loisirs..., volume VIII, op. cit., p. 62.

55. AN, K//160 : pièces manuscrites et imprimées relatives à l'agitation lors des lits de justice de 1787 et 1788 , pièce $n^{\circ} 6,66$, gazette à la main, non signée, « du dimanche 31 août 1788 ».

56. BnF, S.-P. Hardy, Mes loisirs..., volume VIII, op. cit., p. 61.

57. SHD, GR Ya 272, ordre pour l'arrivée de Leurs Majestés à Paris, le 21 janvier 1782.

58. SHD, GR Ya 272, ibid.

59. SHD, GR Ya 272, ibid.

60. AN, 154, AP II, 109, cité par Jean Chagniot (1985:65).

61. E.M.L., Le Parterre justifié ou précis historique et réflexions sur la représentation du 26 décembre 1787, à Londres, 1788, p. 8.

62. Devise gravée sur les canons de Louis XIV signifiant [la force est] le dernier argument des rois.

63. BnF, Lettre de Desbrugnières, inspecteur de police, à son confrère d'Agoult, capitaine aux gardes françaises.

64. Cette expression fut originellement employée par René Héron de Villefosse pour désigner le Palais Royal durant la Révolution (L'Anti Versailles ou le Palais-Royal de Philippe Égalité, Paris, Jean 
Dullis, 1974) puis reprise par Jean Chagniot dans Paris et l'armée au XVIII siècle (p. 28) de façon plus générale pour désigner Paris comme le centre potentiel d'un contre-pouvoir.

65. L.-S. Mercier, Tableau de Paris, tome sixième, édition d'Amsterdam, 1783, p. 201.

66. AN, K//160 : pièces manuscrites et imprimées relatives à l'agitation lors des lits de justice de 1787 et 1788 , pièce $n^{\circ} 6,64$, gazette à la main, non signée, « du 30 août 1788 ».

67. F.-A.-A. de la Chesnaye des Bois, Dictionnaire militaire portatif contenant tous les termes propres à la guerre, tome premier, Paris, chez Duchesne, 1758, p. 166.

68. A.-C. Tardieu de Maleissye, Mémoires d'un officier aux Gardes-françaises, Paris, E. Plon, 1887, p. 16.

69. SHD, GR Ya 272, ordre du 16 mai 1789.

70. BnF, S.-P. Hardy, Mes loisirs..., volume VIII, op. cit., p. 307.

71. BnF, S.-P. Hardy, Mes loisirs..., volume VIII, op. cit., p. 362.

72. J. Menou, Rapport sur l'organisation de la Garde nationale soldée, parisienne, fait à l'Assemblée nationale, dans la séance du 3 août 1791, au nom des comités militaires et de la constitution, Paris, Imprimerie nationale, 1791, p. 10.

73. J.-A.-H. de Guibert, De la force publique considérée dans tous ses rapports, Paris, Didot, 1790.

74. Loi du 28 août 1791, relative à la nouvelle organisation de la Garde nationale soldée, titre premier, article III.

\section{RÉSUMÉS}

Le régiment des Gardes-françaises était à la fin de l'Ancien Régime l'une des principales unités responsables du maintien de l'ordre à Paris. L'étude de la dimension spatiale de l'action du régiment (implantations, trajets des patrouilles et tactiques) renseigne sur la perception de l'espace parisien et de sa population par le pouvoir royal. La proximité du régiment avec ce pouvoir constitue son originalité et le distingue des unités de police active telles que le Guet, placées sous l'autorité du Châtelet. Les Gardes-françaises, qui constituaient donc un œil et un bras armé du pouvoir royal à Paris, n'obéissaient pas seulement à des exigences d'intérêt public, mais ils étaient également soumis à des considérations d'ordre politique qui se traduisaient par une occupation stratégique de l'espace.

The regiment of the Gardes-françaises was one of the major forces in charge of riot control in Paris at the end of the Ancien Régime. The study of the urban and human spatial logics of the regiment - distribution within the city, patrol routes and tactics - are useful to understand the perception of the Parisian space and its population by the monarchy. The proximity of this regiment with the King constitutes its uniqueness and distinguishes it from other police units as the Guet, placed under the authority of the Châtelet. Thus, the Gardes-françaises, who were both an eye and an armed wing of the royal power in Paris, were not only subjected to the public interest but they were also dependent on political issues that were spatially visible through a strategic occupation of space. 
INDEX

Thèmes: Carnets de recherches

Keywords : Army, Riot Control, Gardes-françaises, Ancien Régime

Mots-clés : Armée, maintien de l'ordre, Gardes-françaises, Ancien Régime

\section{AUTEUR}

\section{CLÉMENT MONSEIGNE}

Diplômé d'un Master 2 d'Histoire des mondes modernes et contemporains à l'Université Bordeaux Montaigne.

clement.monseigne[at]gmail.com 